\title{
Emergence and evolution of multiple clusters of attracting agents
}

\author{
Dirk Aeyels, Filip De Smet \\ SYSTeMS Research Group, Dept. of Electrical Energy, Systems and Automation, \\ Ghent University, Technologiepark Zwijnaarde 914, 9052 Zwijnaarde, Belgium
}

\begin{abstract}
We investigate a multi-agent system with a behavior akin to the cluster formation in systems of coupled oscillators. The saturating attractive interactions between an infinite number of non-identical agents, characterized by a multimodal distribution of their natural velocities, lead to the emergence of clusters. We derive expressions that characterize the clusters, and calculate the asymptotic velocities of the agents and the critical value for the coupling strength under which no clustering can occur. The results are supported by mathematical analysis.

For the particular case of a symmetric and unimodal distribution of the natural velocities, the relationship with the Kuramoto model of coupled oscillators is highlighted. While in the generic case the emergence of a cluster corresponds to a second-order phase transition, for a specific choice of the natural velocity distribution a first-order phase transition may occur, a phenomenon recently observed in the Kuramoto model. We also present an example for which the clustering behavior is quantitatively described in terms of the coupling strength.
\end{abstract}

As an illustration of the potential of the model, we discuss how it applies to the dynamic process of opinion formation.

Pacs numbers: $64.60 . \mathrm{Cn}$, 89.75.Fb, 05.45.Xt

Key words: multi-agent systems; phase-transition; self-organization; Kuramoto model; clustering

Published in: Physica D: Nonlinear Phenomena, Vol. 239, No 12, 1026-1037, June, 2010

DOI: $10.1016 /$ j.physd.2010.02.012 


\section{Introduction}

In $[1,2,8]$ we introduced a model representing a system of (a finite number of) mutually attracting, non-identical agents; each agent has a natural velocity, which governs its behavior in the absence of other agents. The agents are attracted to other agents by a saturating interaction function, modulated by a coupling strength, which plays a crucial role in the behavior of the system: for high values, distances between agents remain bounded; for lower values, several clusters arise, each with its characteristic velocity.

The model is relevant for multi-agent systems characterized by a dispersion of the parameters of the individual agents and attractive forces trying to synchronize the behavior of the agents. Examples include animal swarms [23, 6, 17, 8], opinion formation $[12,26,5,9]$, systems of coupled oscillators $[25,16]$.

Research on swarming usually deals with the behavior and/or formation of a single cluster (see e.g. [21]), although the coexistence of multiple clusters is also considered [13]. Regarding opinion formation, in addition to focusing on consenus reaching [12], or considering multiple clusters [9], there are some models that - inspired by the Ising model - consider a polarization into two opposite opinions $[5,26]$. In systems of coupled oscillators one may investigate the synchronization of a single cluster [14], but most models allow the existence of multiple clusters. One distinguishes between phase clustering $[19,10]$ and frequency clustering [18], depending on whether a cluster is characterized by identical phases or identical frequencies. The type of clustering that we will consider in this paper corresponds with frequency clustering.

Due to the complexity and richness of the dynamics of some of these models, analytical results often relate to the existence and local stability properties of some of their solutions $[10,27,3]$, while exploration of the parameter space is usually done by simulations $[11,15]$. The model proposed in $[1,2]$ may be considered a simplification of the Kuramoto model of coupled oscillators, retaining its (frequency) clustering behavior, with an increased potential for developing analytical results. Furthermore, it may be relevant for applications that are not related to coupled oscillators, as we have illustrated in [8] for swarming and opinion formation, and in [2] for compartmental systems.

In this paper we investigate how the results of $[1,2]$ may be recast for an infinite number of agents. We rewrite the conditions for clustering in terms of the distribution of the natural velocities of the agents, and describe how these newly obtained conditions determine the clustering behavior and its dependence on the coupling strength.

In the next section we recall and explain the results of $[1,2]$. In sections 3 and 4 we propose a reformulation of the main result of $[1,2]$ for an infinite 
number of agents. Under some additional assumptions it is proven (see section A of the appendix) that these conditions indeed characterize the clustering behavior in the infinite case. In section 5 we present an example and we show that the cluster formation is remarkably similar to the emergence of partial synchronization in the Kuramoto model. After discussing potential extensions of the model, we illustrate its relation with the process of opinion formation.

\section{A finite number of agents: preliminary results}

The model from $[1,2]$ is described by the following differential equations:

$$
\dot{x}_{i}(t)=b_{i}+\frac{1}{N} \sum_{j=1}^{N} f\left(x_{j}(t)-x_{i}(t)\right),
$$

for $i$ in $\{1, \ldots, N\}$ and $t$ in $\mathbb{R}$, with $N>1$. The function $f$ is odd, nondecreasing, Lipschitz continuous and there exists a $d>0$ such that

$$
f(x)=F, \quad \forall x \geq d .
$$

The dispersion of the natural velocities $b_{i}$ and the attractive interaction (represented by $f$ ) leads to the formation of different clusters. The clusters consist of agents for which the differences in natural velocities are sufficiently small such that the attractive interactions keep them together, while the differences in natural velocities between the clusters is too large with respect to the attraction.

For $n \in \mathbb{N}_{0}$ denote by $I_{n}$ the set $\{1, \ldots, n\}\left(I_{0}=\emptyset\right)$ and let $G=\left(G_{1}, \ldots, G_{M}\right)$ be an ordered partition of $I_{N}$. For convenience we will assume that $b_{1} \leq \cdots \leq$ $b_{N}$. Let $G_{k}^{p}\left(p \in I_{\left|G_{k}\right|-1}\right.$, with $\left|G_{k}\right|$ denoting the number of elements in $\left.G_{k}\right)$ denote the subset containing the largest $p$ numbers in $G_{k}$ and set $\bar{G}_{k}^{p}=G_{k} \backslash G_{k}^{p}$.

For a non-empty set $S \subset I_{N}$ and a vector $w$ denote by $\langle w\rangle_{S}$ the average of $w$ over all elements in $S$ :

$$
\langle w\rangle_{S}=\frac{1}{|S|} \sum_{i \in S} w_{i}
$$

In $[1,2]$ a solution $x$ of (1) is said to exhibit clustering behavior with respect to $G$ if

- the distances between agents in the same cluster remain bounded (i.e. $\left|x_{i}(t)-x_{j}(t)\right|$ is bounded for all $i, j \in G_{k}$, for any $k \in I_{M}$, for $t \geq 0$ );

- after some positive time $T$, the distances between agents in different clusters are at least $d$ and grow unbounded with time; 
- the agents are ordered according to their membership to a cluster: $k<l \Rightarrow$ $x_{i}(t)<x_{j}(t), \forall i \in G_{k}, \forall j \in G_{l}, \forall t \geq T$.

This is the definition of clustering behavior for finite $N$; for an infinite number of agents we will introduce an adapted version.

We recall the following results from [2].

Theorem 1 The inequalities

$$
\begin{aligned}
& \langle b\rangle_{G_{k+1}}-\langle b\rangle_{G_{k}}>\left(\left|G_{k+1}\right|+\left|G_{k}\right|\right) \frac{F}{N}, \quad \forall k \in I_{M-1}, \\
& \langle b\rangle_{G_{k}^{p}}-\langle b\rangle_{\bar{G}_{k}^{p}} \leq\left|G_{k}\right| \frac{F}{N}, \quad \forall p \in I_{\left|G_{k}\right|-1}, \quad \forall k \in I_{M},
\end{aligned}
$$

are necessary and sufficient conditions for clustering behavior with respect to $G$ of all solutions of the system (1).

The formulation of (2b) is slightly different from [2], but it is equivalent under the assumption on the order of the $b_{i}$-values, as is easily verified.

The independence of initial conditions of the clustering behavior follows immediately by observing that the distance in the state space between two solutions $x$ and $x^{*}$ of (1) is non-decreasing: it can be verified (see [2]) that $\frac{\mathrm{d}}{\mathrm{d} t}\left\|x(t)-x^{*}(t)\right\| \leq 0$, for all $t \in \mathbb{R}$. (See also remark 5 later on.)

Using the independence of initial conditions, one derives that the cluster structure $G$ follows the order of the $b_{i}$-values: if $i_{1} \in G_{k_{1}}, i_{2} \in G_{k_{2}}$ and $k_{1}<k_{2}$, then $b_{i_{1}}<b_{i_{2}}$. This is implied by the fact that, for an initial condition with $x_{1}(0) \leq \cdots \leq x_{N}(0)$, this order will be preserved for $t \geq 0$, since whenever $x_{i_{1}}(t)=x_{i_{2}}(t)$, then $\dot{x}_{i_{1}}(t)-\dot{x}_{i_{2}}(t)=b_{i_{1}}-b_{i_{2}}$, as follows from the system equations. It follows also that, if $i_{1}$ and $i_{3}$ belong to the same cluster, with $b_{i_{1}} \leq b_{i_{2}} \leq b_{i_{3}}$ for some $i_{2} \in I_{N}$, then $i_{2}$ also belongs to this cluster.

Theorem 2 For every $b \in \mathbb{R}^{N}$ and every $F>0$, there exists a unique ordered set partition $G$ of $I_{N}$, such that (2) holds.

This theorem was proven (see [2]) by noticing that for high values of $F$ the equations (2) are satisfied for the choice $G=\left(I_{N}\right)$, and by proving that, with $F$ continuously decreasing until (2) ceases to hold at some value $F^{\prime}$, one can switch to another cluster structure $G^{\prime}$ for which (2) is satisfied in the interval $\left[F^{\prime \prime}, F^{\prime}\right)$ for some $F^{\prime \prime}<F^{\prime}$. The argument can be repeated until $F$ equals zero, resulting (in the generic case) in $N$ disjoint intervals for $F$, each corresponding to a different cluster structure. 
Define the values $v_{k}$ as follows:

$$
v_{k} \triangleq\langle b\rangle_{G_{k}}+\frac{F}{N}\left(\sum_{k^{\prime}>k}\left|G_{k^{\prime}}\right|-\sum_{k^{\prime}<k}\left|G_{k^{\prime}}\right|\right) .
$$

It is easy to verify (see [2]) that, for a solution $x$ of (1) exhibiting clustering behavior with respect to $G, v_{k}$ is the average velocity over the cluster $G_{k}$ for sufficiently large $t$. The following theorem states (under some additional conditions) that it is also the asymptotic velocity of the individidual agents in $G_{k}$.

Theorem 3 Assume that $f$ is increasing in the interval $(-d, d)$, and consider a solution $x$ of (1) exhibiting clustering behavior with respect to the cluster structure $G$. Assume that the inequalities (2b) are all satisfied with strict inequality signs. Then, for each $k \in I_{M-1}$, and for all $i, j \in G_{k}$,

$$
\begin{aligned}
& \lim _{t \rightarrow+\infty} x_{i}(t)-x_{j}(t) \text { exists and is independent of } x(0), \\
& \lim _{t \rightarrow+\infty} \dot{x}_{j}(t) \text { exists and equals } v_{k} .
\end{aligned}
$$

The behavior of solutions of (1) can be summarized as follows. The attracting interconnection structure causes a shift for each agent from the natural velocity $b_{i}$ to the asymptotic velocity $v_{k}$ (with $i \in G_{k}$ ), but does not alter the order of the velocities, as determined by the parameters $b_{i}$. After some finite time agents in different clusters will be separated over a distance of at least $d$, and their interaction will equal the saturation value $\pm F$. The distances between agents belonging to the same cluster will converge, and the asymptotic velocity of these agents is therefore equal to the average velocity $v_{k}$ of the agents in $G_{k}$.

\section{An infinite number of agents}

\subsection{The model}

From now on we assume that the natural velocities $b$ are chosen randomly from a continuous distribution with density function $g$ with

$$
\int_{-\infty}^{+\infty} g(b) \mathrm{d} b=1 .
$$


Extending the model (1) to an infinite number of agents, we propose that the dynamics are governed by the following partial differential equation:

$$
\frac{\partial x}{\partial t}(b, t)=b+\int_{-\infty}^{+\infty} g\left(b^{\prime}\right) f\left(x\left(b^{\prime}, t\right)-x(b, t)\right) \mathrm{d} b^{\prime},
$$

for all $b, t$ in $\mathbb{R}$, where we assume $x(b, t)$ to be continuous in $b$ to guarantee that the integral is well-defined. The interaction function has the same properties as before: $f$ is odd, non-decreasing, and Lipschitz continuous, but the saturation property is relaxed:

$$
\lim _{x \rightarrow \pm \infty} f(x)= \pm F .
$$

This relaxation has also been discussed in a previous paper [8].

The behavior of the model is illustrated in Fig. 1, where $f$ is defined as

$$
f(x) \triangleq \begin{cases}x, & |x| \leq F \\ \frac{x}{|x|} F, & |x|>F\end{cases}
$$

with $F$ equal to $\frac{5}{3}$. The density function $g$ is defined as

$$
g(b) \triangleq \frac{4}{7 \sqrt{\pi}}\left(\exp \left(-(b-1)^{2}\right)+\frac{3}{4} \sqrt{2} \exp \left(-2(b+1)^{2}\right)\right),
$$

for all $b$ in $\mathbb{R}$. The picture on the left hand side shows the evolution of $x(b, t)$ as a function of $t$ for some fixed values of $b$, the picture on the right hand side shows $x(b, t) / t$ as a function of $b$ for some fixed values of $t$, together with its limit function $\lim _{t \rightarrow \infty} x(b, t) / t$ (thick line). As can be inferred from both pictures, two clusters emerge. (See also the illustration in section 4.3 later on.) In Fig. 1(a) there are two sets of two agents (asymptotically) moving at the same velocity. In Fig. 1(b) the two clusters are represented by the intervals where $\lim _{t \rightarrow \infty} x(b, t) / t$ is constant.

To investigate the system (1), the approach in [2] was based on the existence of an initial condition such that all clusters are separated over a distance $d$ for all $t \geq 0$. This approach is not possible to prove results for (3) similar to the finite $N$ case, since such an initial condition may not exist. On the one hand this may be a consequence of the presence of an infinite number of clusters. On the other hand, a cluster might have an infinite size, i.e. since a cluster may contain an infinite number of agents, it is possible that (at a fixed time instant) there is no upper bound for the distance between pairs of agents from the same cluster. A related difficulty is the possibility that $x(b, t)$, for a fixed $t$, grows unbounded with $b$, possibly causing the transient time (towards the 'clustering regime') to become infinitely large ${ }^{1}$.

1 Consider e.g. the initial condition where $x(b, 0)=-b^{3}$, for all $b$ in $\mathbb{R}$. Then, for $b \gg F$, the time required for agents with natural velocities $-b$ and $b$ to achieve the 


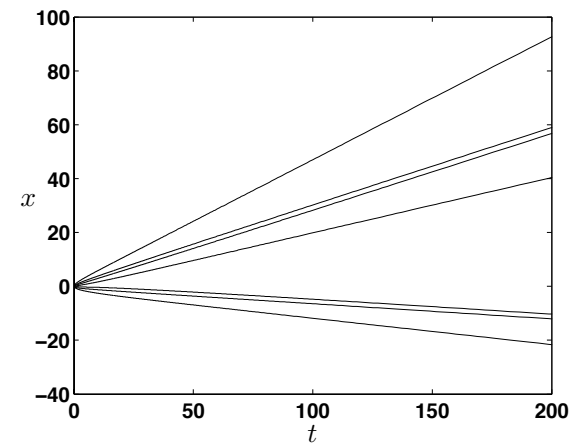

(a)

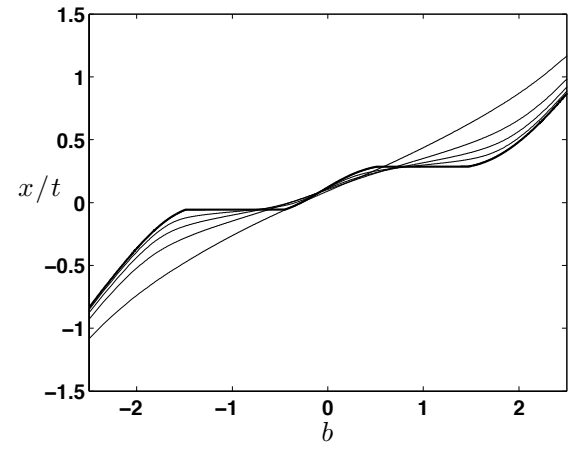

(b)

Fig. 1. The behavior of (3) is shown by plotting $x(b, t)$ as function of $t$ for fixed values of $b$ (Fig. (a), $b \in\{-1.60,-1.05,-0.58,0.20,0.80,1.27,1.96\}$ ), and $x(b, t) / t$ as a function of $b$ for fixed values of $t(t \in\{2.35,6.4,14.4,37.7\})$, with the limit function $v$ shown with a thick line (Fig. (b)). The results were obtained by simulating the finite model (1) with $N=1000$ (with $b_{i}=G^{-1}\left(\left(i-\frac{1}{2}\right) / N\right.$ ) where $G$ is the cumulative density function) using the Euler method with a time step equal to 0.01.

However, with some restrictions imposed on the interaction function $f$ and on the initial condition, we are still able to obtain analytical results. In the next section we apply a heuristic approach to rewrite the conditions (2) for an infinite number of agents, and we present our main theorem, the proof of which is given in section A of the appendix.

\subsection{Cluster structure}

We assume that the property that agents with equal natural velocities belong to the same cluster, which is valid for the finite case, also holds for the infinite case: we may therefore characterize the clusters by sets of $b$-values. Because of the order preservation of the cluster structure with respect to the natural velocities, these sets are convex (or can be extended to a convex set if some natural velocities are not present in the system ${ }^{2}$ ), and therefore they are either singletons or intervals of non-zero length. Consequently each cluster constitutes a well-defined fraction of the population (equal to the integral of $g$ over the corresponding interval). A singleton corresponds to a zero fraction, while a cluster associated with an interval represents a non-zero fraction of

right order (i.e. $x(b, t) \geq x(-b, t)$ ) is approximately $b^{2}$, and for a density function $g$ with infinite support, there will be no finite time $t$ for which $x(b, t)$ is non-decreasing in $b$ for all $b$ in $\mathbb{R}$.

2 E.g. if $g$ is zero in some interval $\left[b_{1}, b_{2}\right]$, and agents with natural velocities slightly smaller than $b_{1}$ belong to the same cluster as agents with natural velocities slightly larger than $b_{2}$, then the interval associated with this cluster is assumed to also contain $\left[b_{1}, b_{2}\right]$. 
the population. From now on we will not refer to the singletons as clusters. (Notice that for the finite case, any non-empty set of agents corresponds to a non-zero fraction of the population; the use of 'non-zero fraction' terminology when defining clusters extends therefore both to the finite and the infinite case.)

We will denote the intervals of non-zero length, each corresponding to a cluster, by $\mathcal{I}_{k}\left(k \in I_{\mathcal{N}}\right.$, where $\mathcal{N} \in \mathbb{N} \cup\{+\infty\}$ denotes the number of these intervals $\left.{ }^{3}\right)$, and we denote the lower and upper endpoint of $\mathcal{I}_{k}$ by $c_{k}$ and $d_{k}$ respectively. The union of the singletons, i.e. the set of all natural velocities $b$ of agents not belonging to a cluster, will be denoted by $\mathcal{S}$.

We define clustering behavior for the system (3) as follows. A solution $x$ of (3) is said to exhibit clustering behavior with respect to a set of intervals $\mathcal{I}_{k}$ $\left(k \in I_{\mathcal{N}}\right)$ if

- $x\left(b_{1}, t\right)-x\left(b_{2}, t\right)$ is bounded for all $b_{1}$ and $b_{2}$ belonging to a common interval $\mathcal{I}_{k}$ for some $k \in I_{\mathcal{N}}$,

- $\lim _{t \rightarrow+\infty}\left|x\left(b_{1}, t\right)-x\left(b_{2}, t\right)\right|=+\infty$ for all $b_{1}$ and $b_{2}$ (with $\left.b_{1} \neq b_{2}\right)$ not belonging to a common interval $\mathcal{I}_{k}$ for any $k \in I_{\mathcal{N}}$.

For an arbitrary interval $\mathcal{I}$ with endpoints $b_{1}<b_{2}$ (possibly infinite) let $\alpha_{\mathcal{I}}$, defined as

$$
\alpha_{\mathcal{I}} \triangleq \int_{b_{1}}^{b_{2}} g\left(b^{\prime}\right) \mathrm{d} b^{\prime}
$$

denote the fraction of agents with natural velocities in the interval $\mathcal{I}$, and, if $\alpha_{\mathcal{I}} \neq 0$, let $\langle b\rangle_{\mathcal{I}}$ denote the average value of $b$ in the interval $\mathcal{I}$, weighted with $g(b)$ :

$$
\langle b\rangle_{\mathcal{I}} \triangleq \frac{1}{\alpha_{\mathcal{I}}} \int_{b_{1}}^{b_{2}} b^{\prime} g\left(b^{\prime}\right) \mathrm{d} b^{\prime}
$$

Led by the expression for $v_{k}$ in the finite case, we introduce the asymptotic velocity function $v: \mathbb{R} \rightarrow \mathbb{R}$ :

$$
v(b) \triangleq \begin{cases}b-F \alpha_{(-\infty, b)}+F \alpha_{(b,+\infty)}, & b \in \mathcal{S} ; \\ \langle b\rangle_{\mathcal{I}_{k}}-F \alpha_{\left(-\infty, c_{k}\right)}+F \alpha_{\left(d_{k},+\infty\right)}, & b \in \mathcal{I}_{k} \text { for some } k \in I_{\mathcal{N}}\end{cases}
$$

3 The number of intervals $\mathcal{I}_{k}$ is countable, since for each $n \in \mathbb{Z}$ the number of intervals $\mathcal{I}_{k}$ contained in the interval $[n, n+1)$ can be counted by considering the intervals with a length in the interval $\left(\frac{1}{i+1}, \frac{1}{i}\right]$ with $i \in \mathbb{N}_{0}$. For each $i$, this number is finite, since the intervals $\mathcal{I}_{k}$ are disjoint and the interval $[n, n+1)$ has a finite length. Considering all possible values for $i$, each interval $\mathcal{I}_{k}$ contained in $[n, n+1)$ will be counted. Since $\mathbb{R}$ is a countable union of intervals $[n, n+1$ ) (with $n \in \mathbb{Z}$ ), and the number of intervals $\mathcal{I}_{k}$ we haven't counted yet all contain an integer - and are therefore also countable - it follows that the number of intervals $\mathcal{I}_{k}$ is countable. 


\section{Clustering conditions}

In this section we adopt a heuristic approach to formulate conditions for the clustering behavior of the model (3). We present our main theorem, providing more rigorous arguments for the characterization of the clustering behavior, and we discuss the general scenario of the emergence of the clusters with increasing coupling strength.

\subsection{Extending the clustering conditions to the infinite case}

To extend the conditions (2) to the system (3), we apply the following reasoning. For a natural velocity $b^{\prime} \in \mathcal{S}$ we consider (2a) with the two groups of agents $G_{k+1}$ and $G_{k}$ corresponding to the natural velocities in the intervals $\left(b^{\prime}, b^{\prime}+\epsilon\right)$ and $\left(b^{\prime}-\epsilon, b^{\prime}\right)$ respectively, in which we let $\epsilon$ approach zero. The inequality (2a) becomes

$$
\langle b\rangle_{\left(b^{\prime}, b^{\prime}+\epsilon\right)}-\langle b\rangle_{\left(b^{\prime}-\epsilon, b^{\prime}\right)}>\left(\alpha_{\left(b^{\prime}, b^{\prime}+\epsilon\right)}+\alpha_{\left(b^{\prime}-\epsilon, b^{\prime}\right)}\right) F,
$$

or

$$
\frac{\int_{b^{\prime}}^{b^{\prime}+\epsilon}\left(b^{\prime \prime}-b^{\prime}\right) g\left(b^{\prime \prime}\right) \mathrm{d} b^{\prime \prime}}{\int_{b^{\prime}}^{b^{\prime}+\epsilon} g\left(b^{\prime \prime}\right) \mathrm{d} b^{\prime \prime}}-\frac{\int_{b^{\prime}-\epsilon}^{b^{\prime}}\left(b^{\prime \prime}-b^{\prime}\right) g\left(b^{\prime \prime}\right) \mathrm{d} b^{\prime \prime}}{\int_{b^{\prime}-\epsilon}^{b^{\prime}} g\left(b^{\prime \prime}\right) \mathrm{d} b^{\prime \prime}}>\int_{b^{\prime}-\epsilon}^{b^{\prime}+\epsilon} g\left(b^{\prime \prime}\right) \mathrm{d} b^{\prime \prime} F .
$$

For $b^{\prime \prime} \in\left(b^{\prime}-\epsilon, b^{\prime}+\epsilon\right), g\left(b^{\prime \prime}\right)$ may be replaced by $g\left(b^{\prime}\right)+o(1)$ (with $o(1)$ denoting an expression that goes to zero when $\epsilon$ goes to zero), because of the continuity of $g$. This leads to

$$
\frac{\frac{\epsilon^{2}}{2}\left(g\left(b^{\prime}\right)+o(1)\right)}{\epsilon\left(g\left(b^{\prime}\right)+o(1)\right)}-\frac{\left(-\frac{\epsilon^{2}}{2}\right)\left(g\left(b^{\prime}\right)+o(1)\right)}{\epsilon\left(g\left(b^{\prime}\right)+o(1)\right)}>2 \epsilon\left(g\left(b^{\prime}\right)+o(1)\right) F,
$$

or, after dividing by $\epsilon$ and taking the limit $\epsilon \rightarrow 0$,

$$
1 \geq 2 g\left(b^{\prime}\right) F
$$

For $k \in I_{\mathcal{N}}$ we obtain for condition (2a)

$$
\langle b\rangle_{\mathcal{I}_{k}}-\langle b\rangle_{\left(c_{k}-\epsilon, c_{k}\right)}>\left(\alpha_{\mathcal{I}_{k}}+\alpha_{\left(c_{k}-\epsilon, c_{k}\right)}\right) F
$$

leading to

$$
\langle b\rangle_{\mathcal{I}_{k}}-c_{k} \geq \alpha_{\mathcal{I}_{k}} F
$$

Similarly

$$
d_{k}-\langle b\rangle_{\mathcal{I}_{k}} \geq \alpha_{\mathcal{I}_{k}} F .
$$


The equations $(2 \mathrm{~b})$ can be reformulated as

$$
\langle b\rangle_{\left(p, d_{k}\right)}-\langle b\rangle_{\left(c_{k}, p\right)} \leq \alpha_{\mathcal{I}_{k}} F, \quad \forall p \in\left(c_{k}, d_{k}\right), \forall k \in I_{\mathcal{N}}
$$

The conditions on the sets $\mathcal{I}_{k}$ and $\mathcal{S}$, defining the clusters, can then be summarized as

$$
\begin{array}{ll}
1 \geq 2 g(b) F, & \forall b \in \mathcal{S}, \\
\langle b\rangle_{\mathcal{I}_{k}}-c_{k}=\alpha_{\mathcal{I}_{k}} F, & \forall k \in I_{\mathcal{N}}, \\
d_{k}-\langle b\rangle_{\mathcal{I}_{k}}=\alpha_{\mathcal{I}_{k}} F, & \forall k \in I_{\mathcal{N}}, \\
\langle b\rangle_{\left(p, d_{k}\right)}-\langle b\rangle_{\left(c_{k}, p\right)} \leq \alpha_{\mathcal{I}_{k}} F, & \forall p \in\left(c_{k}, d_{k}\right), \forall k \in I_{\mathcal{N}} .
\end{array}
$$

The equations (5b) and (5c) are obtained from previous inequalities together with the result of taking the limits $p \rightarrow c_{k}$ and $p \rightarrow d_{k}$ in (5d). They are equivalent with

$$
\begin{aligned}
& d_{k}-c_{k}=2 \alpha_{\mathcal{I}_{k}} F, \quad \forall k \in I_{\mathcal{N}}, \\
& \frac{c_{k}+d_{k}}{2}=\langle b\rangle_{\mathcal{I}_{k}}, \quad \forall k \in I_{\mathcal{N}},
\end{aligned}
$$

or

$$
\begin{aligned}
\frac{\int_{\mathcal{I}_{k}} g\left(b^{\prime}\right) \mathrm{d} b^{\prime}}{d_{k}-c_{k}} & =\frac{1}{2 F}, \quad \forall k \in I_{\mathcal{N}}, \\
\frac{\int_{\mathcal{I}_{k}} b^{\prime} g\left(b^{\prime}\right) \mathrm{d} b^{\prime}}{\int_{\mathcal{I}_{k}} g\left(b^{\prime}\right) \mathrm{d} b^{\prime}} & =\frac{c_{k}+d_{k}}{2}, \quad \forall k \in I_{\mathcal{N}} .
\end{aligned}
$$

In other words, the average value of the density function $g$ over an interval $\mathcal{I}_{k}$ equals $\frac{1}{2 F}$, and the average value of $b$ over the associated cluster corresponds to the center of the interval $\mathcal{I}_{k}$.

\subsection{Main clustering result}

The relevance of the conditions (5) for the system (3) is established by the following theorem.

Theorem 4 Assume that $f$ is odd, non-decreasing and satisfies $\lim _{x \rightarrow \pm \infty} f(x)=$ $\pm F$, with $F>0$. Furthermore assume that $f$ is twice differentiable with $\frac{\mathrm{d} f}{\mathrm{~d} x}$ unimodal and $\frac{\mathrm{d}^{2} f}{\mathrm{~d} x^{2}}$ bounded. Consider a solution $x$ of $(3)$ with $\inf _{b \in \mathbb{R}} \frac{\partial x}{\partial b}(b, 0) \geq 0$, and $\sup _{b \in \mathbb{R}} \frac{\partial x}{\partial b}(b, 0)<1 / \frac{\mathrm{d} f}{\mathrm{~d} x}(0)$, with $x$ at least twice differentiable.

Then there exist intervals $\mathcal{I}_{k} \subset \mathbb{R}, k \in I_{\mathcal{N}}$ for some $\mathcal{N} \in \mathbb{N} \cup\{+\infty\}$, such that $x$ exhibits clustering behavior with respect to the set of intervals $\mathcal{I}_{k}$. 
Furthermore, the sets $\mathcal{I}_{k}$ and $\mathcal{S} \triangleq \mathbb{R} \backslash \bigcup_{k \in I_{\mathcal{N}}} \mathcal{I}_{k}$ satisfy (5), and, defining $v$ by (4),

$$
\lim _{t \rightarrow+\infty} \frac{\partial x}{\partial t}(b, t)=v(b), \quad \forall b \in \mathbb{R}
$$

The extra assumptions on $f$ may not be necessary, as they do not appear in the finite case, but they simplify the mathematical analysis. The proof is given in section $\mathrm{A}$ of the appendix.

Theorem 4 implies that the conditions (5) are necessary for the associated clustering behavior. They cannot be sufficient, since if $g$ is constant in some interval, then different solutions for the set of intervals $\mathcal{I}_{k}$ satisfying (5) exist (even without making a distinction between open and closed intervals). E.g. assume that $g(b)=g_{0}$ for $b \in\left[b_{1}, b_{2}\right]$, and that $g_{0}=\max (g)$. Then for $F=$ $1 /\left(2 g_{0}\right)$ any set of disjoint intervals $\mathcal{I}_{k}$, with $\mathcal{I}_{k} \subset\left[b_{1}, b_{2}\right]$, satisfies (5) (with $\left.\mathcal{S}=\mathbb{R} \backslash \bigcup_{k \in I_{\mathcal{N}}} \mathcal{I}_{k}\right)$. Taking into account these aspects, Theorem 1 suggests that an adaptation of (5) might exist that - under some extra conditions - represents a sufficient condition for clustering behavior of solutions of the system (3).

For convenience we assume in the remainder of the paper (except for section A of the appendix) that the conditions (5) 'largely' characterize the clustering behavior of solutions of (3), keeping in mind that this may not be true for particular values of $F$, and that (5) does not tell us whether to consider open or closed intervals $\mathcal{I}_{k}$.

\subsection{Cluster growth with increasing coupling strength}

If $g$ has a finite number of local maxima, the creation or disappearance of the clusters can be described as follows. For $F<\frac{1}{2 \max (g)}$ there are no clusters, and then $\mathcal{S}=\mathbb{R}$. For $F \gtrsim \frac{1}{2 \max (g)}$ a cluster arises in an interval $\mathcal{I}_{1}$ containing the value where the maximum of $g$ is attained. When increasing $F$, this cluster increases such that (6) remains satisfied: the average value of $g$ over the interval $\mathcal{I}_{1}$ equals $\frac{1}{2 F}$ and the average value of $b$ over the cluster is the center of $\mathcal{I}_{1}$. If the value of $\frac{1}{2 F}$ reaches another local maximum of $g$ then a new cluster arises, associated with an interval for the natural velocities that contains the value of $b$ for which the local maximum of $g(b)$ is attained. This guarantees that outside the intervals $\mathcal{I}_{k}$ the equations (5a) will hold. In section B.1 of the appendix we provide arguments indicating that for a fixed $p,(5 \mathrm{~d})$ will continue to hold when increasing $F$. Since for a given $p$, the first occurrence of (5d) when increasing $F$ appears for $p=c_{k}$ in (5b) or $p=d_{k}$ in (5c), this suggests that $(5 \mathrm{~d})$ will always hold when applying this procedure. This means that — while increasing $F$ - we only need to consider the equations (6). Of 
course, if two clusters collide (i.e. $c_{k}-d_{k-1} \rightarrow 0$ for some $k$ ) when increasing $F$, they become one cluster. In section B.3 of the appendix we verify that this new cluster still satisfies (6).

This scenario is illustrated in Fig. 2, where the same density function $g$ is considered (dashed line) as before when illustrating the dynamics in Fig. 1:

$$
g(b)=\frac{4}{7 \sqrt{\pi}}\left(\exp \left(-(b-1)^{2}\right)+\frac{3}{4} \sqrt{2} \exp \left(-2(b+1)^{2}\right)\right)
$$

for all $b$ in $\mathbb{R}$. The solid line in Fig. 2 shows the endpoints $c_{k}$ and $d_{k}$ (horizontal axis) of the cluster intervals $\mathcal{I}_{k}$ versus the value of $g_{F} \triangleq 1 /(2 F)$ (vertical axis), which is equal to the average value of $g$ over an interval $\mathcal{I}_{k}$ (see equation (6a)). As an illustration, the dotted lines show the two cluster intervals $\mathcal{I}_{1}$ and $\mathcal{I}_{2}$ for a value of $g_{F}=0.3$.

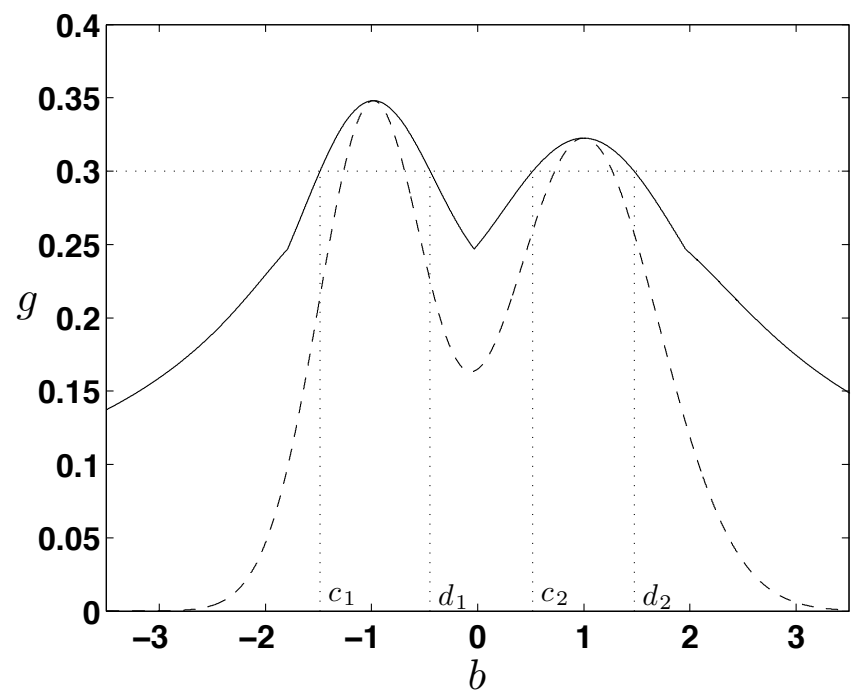

Fig. 2. Density function $g$ (dashed line) and evolution of the cluster(s) (solid line) with varying coupling strength. The dotted lines show the cluster intervals for a value of $g_{F}=0.3$.

Remark 5 In [2, 8] we showed that the clustering behavior of solutions of the system (1) with finite $N$ was independent of the initial condition. Below we show that the extension of the approach from [2, 8] to the system (3) fails.

Consider the solution $x^{*}$ corresponding to the initial condition $x^{*}(b, 0)=0$, $\forall b \in \mathbb{R}$, and let $x$ be an arbitrary solution for which $\int_{-\infty}^{+\infty}(x(b, 0))^{2} g(b) \mathrm{d} b$ is finite. Then the function $V$, defined by

$$
V(t) \triangleq \int_{-\infty}^{+\infty}\left(x(b, t)-x^{*}(b, t)\right)^{2} g(b) \mathrm{d} b, \quad \forall t \in \mathbb{R}
$$


exists for $t=0$ and satisfies

$$
\begin{aligned}
\frac{\mathrm{d} V}{\mathrm{~d} t}(t)=2 \int_{-\infty}^{+\infty} & \left(x(b, t)-x^{*}(b, t)\right) g(b) \mathrm{d} b \\
& \times \int_{-\infty}^{+\infty}\left(f\left(x\left(b^{\prime}, t\right)-x(b, t)\right)-f\left(x^{*}\left(b^{\prime}, t\right)-x^{*}(b, t)\right)\right) g\left(b^{\prime}\right) \mathrm{d} b^{\prime} \\
=-\int_{-\infty}^{+\infty} & \int_{-\infty}^{+\infty}\left(\left(x\left(b^{\prime}, t\right)-x(b, t)\right)-\left(x^{*}\left(b^{\prime}, t\right)-x^{*}(b, t)\right)\right) \\
& \times\left(f\left(x\left(b^{\prime}, t\right)-x(b, t)\right)-f\left(x^{*}\left(b^{\prime}, t\right)-x^{*}(b, t)\right)\right) g(b) g\left(b^{\prime}\right) \mathrm{d} b \mathrm{~d} b^{\prime}
\end{aligned}
$$

$\leq 0$

However, the implication that $V$ is bounded does not imply that the integrand in the definition of $V(t)$, and therefore also $x(b, t)-x^{*}(b, t)$, is bounded in $t$ for any $b$. Consequently, the boundedness of $V$ is not sufficient to conclude that $x^{*}$ and $x$ have the same clustering behavior.

\section{A unimodal and symmetric distribution: relation to the $\mathrm{Ku}$ - ramoto model}

In this section we consider a symmetric and unimodal density function $g$, and we investigate the phase transition associated with the emergence of a cluster. We also compare the behavior of the system (3) with the behavior of the $\mathrm{Ku}$ ramoto model, and in particular we discuss the possibility for the occurrence of a first-order phase transition in both models, under similar conditions. (As in $[20,4]$, we will use the notions of first-order and second-order phase transitions to describe the onset of the cluster formation, even though the system (3) may not correspond to a thermodynamic system.)

\subsection{General scenario: a second-order phase transition}

If $g$ is strictly increasing in $\mathbb{R}^{-}$and strictly decreasing in $\mathbb{R}^{+}$, there will be at most one cluster $\mathcal{I}_{k}(\mathcal{N} \leq 1)$, as can be derived from (6). Indeed, if the corresponding interval is denoted by $\mathcal{I}_{1}$, then equation $(6 \mathrm{~b})$ implies $c_{1}=-d_{1}$ (since it follows that $\langle b\rangle_{\mathcal{I}_{1}}<\frac{c_{1}+d_{1}}{2}$ for $c_{1}+d_{1}>0$, or $\langle b\rangle_{\mathcal{I}_{1}}>\frac{c_{1}+d_{1}}{2}$ for $\left.c_{1}+d_{1}<0\right)$, and $(6 a)$ can be written as

$$
d_{1}=2 F \int_{0}^{d_{1}} g(b) \mathrm{d} b
$$

with at most one non-zero solution for $d_{1}$ for a fixed value of $F$ (as can be inferred from the properties of $g$ ). 
If $g$ is not strictly monotonous in $\mathbb{R}^{-}$and $\mathbb{R}^{+}$, then $g$ is constant in some interval, and we will assume that also in this case there is at most one cluster, associated with the interval $\mathcal{I}_{1}$, with $c_{1}=-d_{1}$ and $d_{1}$ the largest solution to (7). (See also the discussion below Theorem 4.)

If there are no clusters, we will set $\mathcal{I}_{1} \triangleq \emptyset$ and $c_{1}=d_{1} \triangleq 0$ (notice that $d_{1}=0$ is always a solution of (7)) for convenience. Then we can introduce $\alpha_{\mathcal{I}_{1}}$ as an order parameter: $\alpha_{\mathcal{I}_{1}}$ varies between 0 and 1 and corresponds to the fraction of agents that are organized in a cluster.

Assuming that the density function $g$ is three times continuously differentiable in a neighborhood of zero, we can investigate the onset of clustering for small values of $d_{1}$ by the equation (notice that, because of the assumptions on $g$, $g^{\prime}(0)=g^{\prime \prime \prime}(0)=0$ and $\left.g^{\prime \prime}(0) \leq 0\right)$

$$
d_{1}=2 F\left(g(0) d_{1}+\frac{g^{\prime \prime}(0)}{6} d_{1}^{3}+o\left(d_{1}^{4}\right)\right)
$$

resulting in either $d_{1}=0$ and $\alpha_{\mathcal{I}_{1}}=0$ (no clustering, corresponding to the case $F \leq 1 /(2 g(0)))$ or (if $g^{\prime \prime}(0) \neq 0$ )

$$
d_{1} \approx \sqrt{\frac{6}{-g^{\prime \prime}(0)}\left(g(0)-\frac{1}{2 F}\right)} .
$$

Consequently, at the onset of the cluster formation, $\alpha_{\mathcal{I}_{1}}$ can be approximated for $F \geq 1 /(2 g(0))$ as

$$
\alpha_{\mathcal{I}_{1}} \approx \frac{1}{F} \sqrt{\frac{6}{-g^{\prime \prime}(0)}\left(g(0)-\frac{1}{2 F}\right)}
$$

or, since then $F \approx 1 /(2 g(0))$,

$$
\alpha_{\mathcal{I}_{1}} \approx 2 g(0) \sqrt{\frac{6}{-g^{\prime \prime}(0)}\left(g(0)-\frac{1}{2 F}\right)} .
$$

This relation shows that when a cluster emerges around 0 with $g^{\prime \prime}(0)<0$, $\alpha_{\mathcal{I}_{1}}$ grows continuously — but not differentiably — with $F$, indicative of a second-order phase transition.

Example 6 Consider the distribution g, defined by

$$
g(b) \triangleq \frac{1}{2 \sqrt{1+b^{2}}\left(\sqrt{1+b^{2}}+1\right)}, \quad \forall b \in \mathbb{R},
$$


for which

$$
\int_{0}^{d_{1}} g(b) \mathrm{d} b=\frac{1}{2 d_{1}}\left(\sqrt{1+d_{1}^{2}}-1\right)=\frac{d_{1}}{2\left(\sqrt{1+d_{1}^{2}}+1\right)},
$$

and thus the relation between $d_{1}$ and $F$ for $d_{1} \neq 0$ is given by

$$
d_{1}=F \sqrt{1-\frac{2}{F}}
$$

for $F \geq \frac{1}{2 g(0)}=2$. It also follows that

$$
\alpha_{\mathcal{I}_{1}}=\sqrt{1-\frac{2}{F}}
$$

This relation is illustrated in Fig. 3, showing the typical evolution of an order parameter for a second-order phase transition.

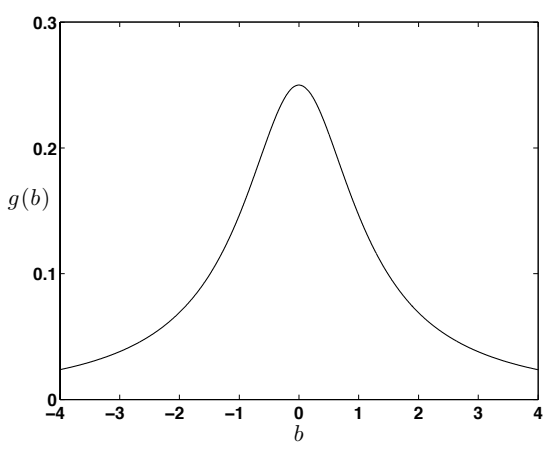

(a)

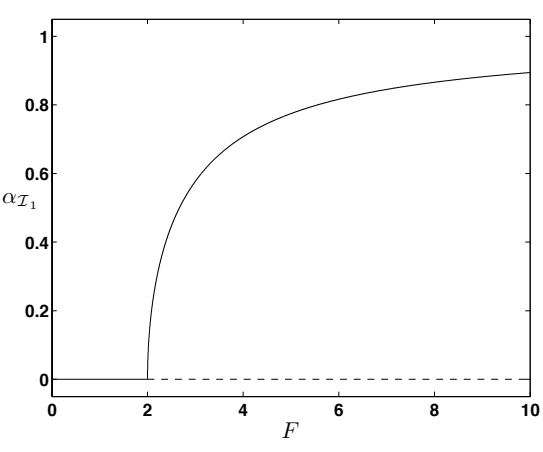

(b)

Fig. 3. Density function $g$ (Fig. (a)) and evolution of the order parameter in terms of $F$ (Fig. (b)) for the given example.

\subsection{Relation to the Kuramoto model}

The emergence of a single cluster in the current model is similar to the development of partial synchronization in the Kuramoto model [14]. This model, introduced by Kuramoto to describe synchronization in systems of coupled oscillators, is represented by the differential equations

$$
\dot{\theta}_{i}(t)=\omega_{i}+\frac{K}{N} \sum_{j=1}^{N} \sin \left(\theta_{j}(t)-\theta_{i}(t)\right),
$$

for $i$ in $\{1, \ldots, N\}$ and $t$ in $\mathbb{R}$, where the natural frequencies $\omega_{i}$ are drawn from a distribution $g$, which is unimodal and symmetric. For an infinite number of oscillators the behavior is either incoherent - oscillators with different natural 
frequencies exhibit different long term average frequencies - or characterized by one synchronized cluster - consisting of oscillators with the same frequency - and the remaining oscillators moving incoherently. An order parameter $r$ is introduced in [14] and is shown to satisfy a self-consistency equation (for details, see [24]), which we write as

$$
K r=2 K \int_{0}^{K r} g(\omega) \sqrt{1-\frac{\omega^{2}}{K^{2} r^{2}}} \mathrm{~d} \omega
$$

emphasizing the similarity with equation (7). There is always the solution $r=0$, corresponding to total incoherence, while for $K$ above the critical value of $K_{\mathrm{c}}=\frac{2}{\pi g(0)}$ a non-zero solution exists, with the corresponding partially synchronized cluster consisting of all oscillators with $\omega \in[-K r, K r]$. For $K \gtrsim K_{\mathrm{c}}$ the product $K r$ can be approximated by (see [24])

$$
K r \approx \sqrt{\frac{8}{-g^{\prime \prime}(0)}\left(g(0)-\frac{2}{K \pi}\right)},
$$

which has a striking resemblance to (8).

For multimodal or asymmetric distributions of the parameters $b$ and $\omega$, we notice some differences in the behavior of the current model and the Kuramoto model:

- Clusters of the model (3) always start in a local maximum of the distribution of the natural velocities, while for the Kuramoto model this is no longer true for a general distribution of the natural frequencies (see e.g. [7]). However, for the Kuramoto model, the clusters still seem to originate 'near' a local maximum, and a high density of oscillators (in the natural frequency space) is still a requirement for clustering. This can be attributed to the different structure of the interactions in both models. Because of the saturation of the interaction in the model (3), it makes no difference for the properties of a particular cluster what the actual values of $b$ are for agents that do not belong to this cluster; the effective interaction will be the same saturation value. However, for the Kuramoto model, the interaction between oscillators will vary with varying $\omega$-values, even if they do not belong to the same cluster. As a result, the size and the location of a cluster in the Kuramoto model will depend on the overall properties of the distribution of the natural frequencies, while for the model (3) only local properties need to be considered.

- Although (3) can (qualitatively) generate the clustering process of the Kuramoto model, other phenomena typical for systems of coupled oscillators such as frequency locking [15] or induction of clusters by resonances [7] are not reproduced by (3), which explains why this model is easier to analyze than the Kuramoto model. This is also related to the previous remark, since 
these phenomena do not depend on local properties only, which makes it harder to investigate them.

\subsection{A first-order phase transition}

In the Kuramoto model (see previous section) there is a first-order phase transition when the distribution of the natural frequencies is uniform [20,4]. In a similar way the system (3) exhibits a first-order phase transition for a distribution $g$ where the maximum is attained in an interval $\mathcal{I}$ (with a uniform distribution as a special case). Denoting by $g_{0}$ the value of $g$ in this interval, a cluster will emerge for $F=1 /\left(2 g_{0}\right)$, containing all agents with natural velocities in $\mathcal{I}$ (possibly excluding the endpoints of $\mathcal{I}$ ). The order parameter $\alpha_{\mathcal{I}_{1}}$ will jump from zero to

$$
\alpha_{\mathcal{I}}=\int_{\mathcal{I}} g\left(b^{\prime}\right) \mathrm{d} b^{\prime}
$$

introducing a discontinuity, which is characteristic for a first-order phase transition. (See Fig. 4.)

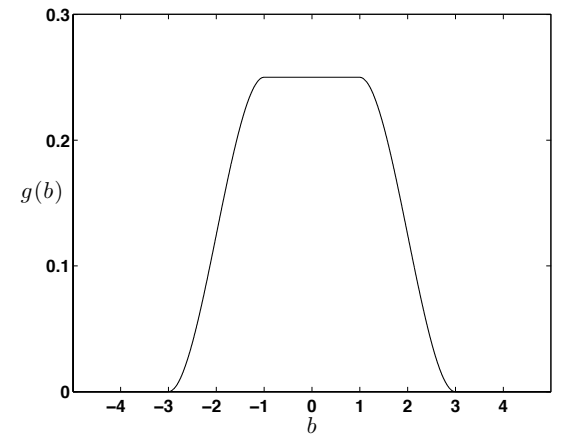

(a)

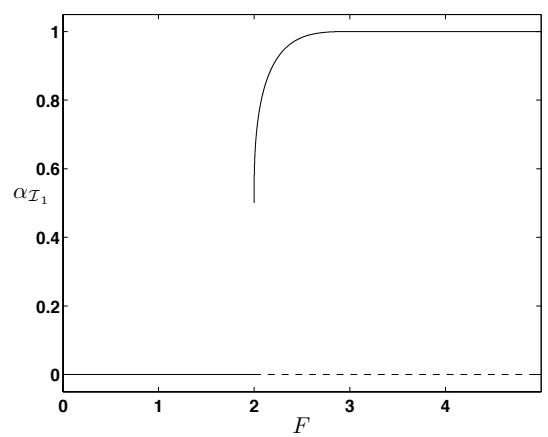

(b)

Fig. 4. Evolution of the order parameter in terms of $F$ (Fig. (b)) for the density function $g$ shown in Fig. (a), indicating a first-order phase transition.

\section{Extensions}

The model (3) can be extended to include weighting factors and a general interaction structure (as has been done for the finite case in [8]), but, except for the observations below, we will not elaborate on this. 


\subsection{Weighting factors}

Introducing a weighting function $\gamma: \mathbb{R} \rightarrow \mathbb{R}_{0}^{+}$(where $\int_{-\infty}^{+\infty} g\left(b^{\prime}\right) \gamma\left(b^{\prime}\right) \mathrm{d} b^{\prime}$ is assumed to be finite), results in the following system:

$$
\frac{\partial x}{\partial t}(b, t)=b+\frac{\int_{-\infty}^{+\infty} g\left(b^{\prime}\right) \gamma\left(b^{\prime}\right) f\left(x\left(b^{\prime}, t\right)-x(b, t)\right) \mathrm{d} b^{\prime}}{\int_{-\infty}^{+\infty} g\left(b^{\prime}\right) \gamma\left(b^{\prime}\right) \mathrm{d} b^{\prime}} .
$$

Obviously, an appropriate redefinition of $g$ leads again to (3), and the mathematical treatment is not affected by the introduction of weighting factors.

\subsection{General coupling structure}

Introducing a dependency of the interaction on the natural velocities of the interacting agents results in:

$$
\frac{\partial x}{\partial t}(b, t)=b+\int_{-\infty}^{+\infty} g\left(b^{\prime}\right) f\left(b, b^{\prime}, x\left(b^{\prime}, t\right)-x(b, t)\right) \mathrm{d} b^{\prime} .
$$

If $f\left(b, b^{\prime}, x\right)$ is symmetric in $b$ and $b^{\prime}$, i.e. $f\left(b_{1}, b_{2}, x\right)=f\left(b_{2}, b_{1}, x\right), \forall b_{1}, b_{2}, x \in$ $\mathbb{R}$, then results from [8] may be used to obtain conditions similar to (5) for the emerging clusters. However, a different approach from the one in this paper will be needed to support these conditions by mathematical analysis. An important point implied by the results from [8] consists of the fact that $v$ will not necessarily be non-decreasing, and agents with small natural velocities may have larger asymptotic velocities than agents with larger natural velocities.

\section{Opinion formation}

In this section we show how the model (3) may apply to opinion formation, with on the one hand a wide range of possible outcomes, and on the other hand the potential to obtain these outcomes by analytical means.

An opinion on a particular matter is represented by a real number, with zero corresponding to a neutral position. We consider a large number of individuals taking part in a discussion. (For smaller numbers of participants the approach should be based on the finite model (1).) Each individual has his own opinion, which may evolve in time as a result of the discussion with the other participants. Since opinions cannot grow unbounded, $x(b, t)$ in $(3)$ seems not to be appropriate as a quantity to represent an opinion. Instead we will take the time derivatives $y=\frac{\partial x}{\partial t}$ as a measure of someone's opinion. (Although in 
[22], in a similar model, the value of $x$ was chosen to represent an opinion, we believe that $y$ is a better choice as it is guaranteed to be bounded in time.) Furthermore we assign weights $\gamma(b)$ to the participants, as introduced in the previous section. Assuming that the weights are normalized as follows:

$$
\int_{-\infty}^{+\infty} \mathrm{d} b g(b) \gamma(b)=1
$$

the equations for $y$ can be written as (assuming $x(b, 0)=0$, for all $b$ in $\mathbb{R}$ )

$$
y(b, t)=b+\int_{-\infty}^{+\infty} \mathrm{d} b^{\prime} g\left(b^{\prime}\right) \gamma\left(b^{\prime}\right) f_{i j}\left(\int_{0}^{t}\left(y\left(b, t^{\prime}\right)-y\left(b^{\prime}, t^{\prime}\right)\right) \mathrm{d} t^{\prime}\right),
$$

for all $b$ in $\mathbb{R}$.

In general each participant starts with an 'a priori' opinion $b$, while with time and through interaction, different groups may be formed, each characterized by a final opinion $v(b)$. The pressure to reach a decision, or the tendency to adapt one's opinion by paying attention to each other's arguments is reflected by the interaction strength $F$.

With $y(b, t)$ representing the opinion of an individual at time $t$, the absolute value of the integral $\int_{0}^{t}\left(y\left(b, t^{\prime}\right)-y\left(b^{\prime}, t^{\prime}\right)\right) \mathrm{d} t^{\prime}$ may reflect the level of disagreement accumulated over time, or the amount of discussions taking place between participants with a priori opinions $b$ and $b^{\prime}$, proportional with time and with difference in opinion. The weight function $\gamma(b)$ expresses the tendency of a participant with a priori opinion $b$ to discuss with and to convince other participants.

As an example we consider a Gaussian distribution for the a priori opinions:

$$
g(b)=\frac{1}{\sqrt{2 \pi}} e^{-\frac{b^{2}}{2}}
$$

and we compare constant weights:

$$
\gamma_{1}(b)=1
$$

for all $b$ in $\mathbb{R}$, (equivalent to not considering any weights) with the following weight distribution:

$$
\gamma_{2}(b)=\frac{1+2 b^{2}}{\int_{-\infty}^{+\infty} \mathrm{d} b^{\prime} g\left(b^{\prime}\right)\left(1+2{b^{\prime}}^{2}\right)}=\frac{1}{3}\left(1+2 b^{2}\right) .
$$

for all $b$ in $\mathbb{R}$. This latter weight distribution reflects that people with extreme opinions are usually more inclined to try to convince other people of their beliefs. 
The resulting evolution of the clusters is shown in Fig. 5, similarly as in Fig. 2: besides the modified density functions $\gamma_{1} g$ and $\gamma_{2} g$ (dashed lines), the boundaries of the associated clusters are represented on the horizontal axis (solid lines) with the corresponding value of $g_{F} \triangleq 1 /(2 F)$ shown on the vertical axis. The modified distribution $\gamma_{1} g$ is unimodal and will therefore result in the emergence of a single cluster, while $\gamma_{2} g$ is bimodal (and symmetric around zero) and will give rise to two clusters, which will merge and form one cluster only if the value of $F$ is sufficiently large (and $g_{F}$ is sufficiently small). This new cluster will be smaller than the corresponding cluster for the weight function $\gamma_{1}$, but the difference in cluster size goes to zero with increasing $F$. We conclude that the influence of the weight function $\gamma$ is only relevant for intermediate values of $F$, for which $\gamma_{1}$ may result in a substantial agreement, while $\gamma_{2}$ leads to either no consensus at all or a polarization into two opposing opinions.

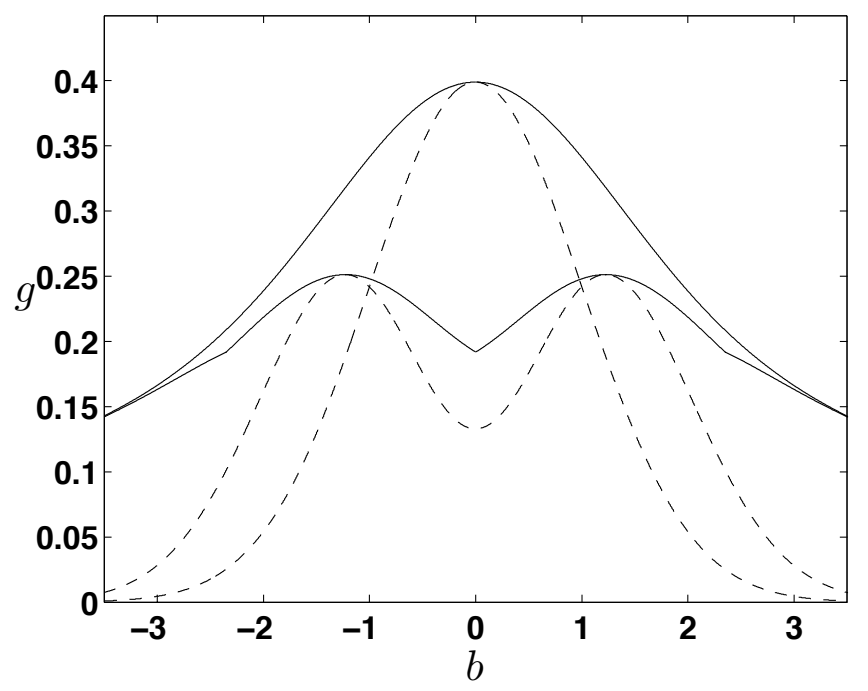

Fig. 5. Evolution of the cluster growth for the modified density functions $\gamma_{1} g$ (unimodal) and $\gamma_{2} g$ (bimodal). Both modified density functions are plotted with dashed lines, the corresponding cluster boundaries are shown with solid lines.

Depending on the coupling strength $F$ and the shape of the modified density function $\gamma g$, different outcomes are possible, from total heterogeneity with each individual having its own opinion, over a combination of clusters of participants with a common opinion and individuals with their own opinion, to total consensus (if $g$ has finite support).

The model (11) distinguishes itself from other existing models (for an overview, see [12]) not only by its potential for generating a wide range of outcomes, but also by its analytical tractability, allowing us to calculate the final opinions. 


\section{Conclusion}

We have considered the continuum limit in a multi-agent clustering model for which the behavior of the agents is determined by non-identical preferential velocities and a saturating attraction between the agents. There are an infinite number of agents, represented by a multimodal distribution for the natural velocities. We have investigated the long term behavior of the model, thereby deriving conditions characterizing the emerging cluster structure. The velocities of the agents converge to limit values, which can be represented by a continuous function of the natural velocities. The intervals where this function is constant correspond to the different clusters; each cluster is associated with a single asymptotic velocity value.

With increasing coupling strength, clusters arise at local maxima of the density function that defines the natural velocities. When the coupling strength is increased further, the clusters grow, and in case two (or more) clusters collide, they merge and form a new cluster. The evolution of the clusters can be easily described analytically.

The clustering behavior of the model is similar to the behavior of the Kuramoto model of coupled oscillators. As in the Kuramoto model clusters appear if the product of density and coupling strength is (locally) high enough. Furthermore, in both models the emergence of a cluster generally resembles a second-order phase transition, but a well-chosen distribution of the natural velocities may lead to behavior characteristic of a first-order phase transition. However, in the clustering model the location and the size of a cluster only depend locally on the density function defining the natural velocities, while for the Kuramoto model there is a dependence on the entire distribution of the natural frequencies.

In addition to similarities with the dynamic behavior of the Kuramoto model, the clustering model may be relevant for applications not related to coupled oscillators, such as opinion formation. Depending on the coupling strength and on the distribution of the a priori opinions, different outcomes are possible, ranging from different opinions for all individuals to a complete consensus.

\section{Acknowledgments}

This paper presents research results of the Belgian Programme on Interuniversity Attraction Poles, initiated by the Belgian Federal Science Policy Office. The scientific responsibility rests with its authors. 
During this research Filip De Smet was supported by a Ph.D. fellowship of the Research Foundation - Flanders (FWO).

\section{A Proof of Theorem 4}

We first show that both $\frac{\partial x}{\partial b}$ and $\frac{\partial^{2} x}{\partial t \partial b}$ are non-negative in $\mathbb{R} \times \mathbb{R}^{+}$.

Non-negativeness of $\frac{\partial x}{\partial b}(b, t)$

Taking the partial derivative to $b$ in $(3)$ results in

$$
\frac{\partial^{2} x}{\partial t \partial b}(b, t)=1-\frac{\partial x}{\partial b}(b, t) \phi(b, t)
$$

with

$$
\phi(b, t) \triangleq \int_{-\infty}^{+\infty} g\left(b^{\prime}\right) \frac{\mathrm{d} f}{\mathrm{~d} x}\left(x\left(b^{\prime}, t\right)-x(b, t)\right) \mathrm{d} b^{\prime} .
$$

The differential equation (A.1) can be solved for $\frac{\partial x}{\partial b}(b, t)$ as

$$
\frac{\partial x}{\partial b}(b, t)=\exp \left(-\int_{0}^{t} \phi\left(b, t^{\prime}\right) \mathrm{d} t^{\prime}\right)\left(\int_{0}^{t} \exp \left(\int_{0}^{t^{\prime}} \phi\left(b, t^{\prime \prime}\right) \mathrm{d} t^{\prime \prime}\right) \mathrm{d} t^{\prime}+\frac{\partial x}{\partial b}(b, 0)\right)
$$

and since $\frac{\partial x}{\partial b}(b, 0) \geq 0$, for all $b$ in $\mathbb{R}, \frac{\partial x}{\partial b}(b, t)$ is non-negative for all $(b, t)$ in $\mathbb{R} \times \mathbb{R}^{+}$.

\section{Non-negativeness of $\frac{\partial^{2} x}{\partial t \partial b}(b, t)$}

By the choice of the initial condition it follows from (A.1) that $\frac{\partial^{2} x}{\partial t \partial b}(b, 0) \geq \epsilon$, for all $b$ in $\mathbb{R}$, for some $\epsilon>0$, since $\phi(b, t) \leq \frac{\mathrm{d} f}{\mathrm{~d} x}(0)$. Assume that $\frac{\partial^{2} x}{\partial t \partial b}(b, t)$ is negative for some $b \in \mathbb{R}$ and some $t>0$ and define $t_{1}$ as

$$
t_{1} \triangleq \inf \left\{t \in \mathbb{R}^{+}: \exists b \in \mathbb{R}, \frac{\partial^{2} x}{\partial t \partial b}(b, t)<0\right\} .
$$

For $t \in\left[0, t_{1}\right]$ the inequality $\frac{\partial^{2} x}{\partial t \partial b}(b, t) \geq 0$ holds for all $b \in \mathbb{R}$, and thus $b^{\prime}-b$ has the same sign as

$$
\frac{\partial}{\partial t}\left(x\left(b^{\prime}, t\right)-x(b, t)\right)=\int_{b}^{b^{\prime}} \frac{\partial^{2} x}{\partial t \partial b}\left(b^{\prime \prime}, t\right) \mathrm{d} b^{\prime \prime} .
$$

It follows that for fixed $b$ and $b^{\prime}, \frac{\mathrm{d} f}{\mathrm{~d} x}\left(x\left(b^{\prime}, t\right)-x(b, t)\right)$ and therefore also $\phi(b, t)$ are non-increasing in $t$ in the interval $\left[0, t_{1}\right]$. 
From (A.1) and (A.2) one can derive that

$$
\begin{aligned}
\frac{\partial}{\partial t}\left(\exp \left(\int_{0}^{t} \phi\left(b, t^{\prime}\right) \mathrm{d} t^{\prime}\right)\right. & \left.\frac{\partial^{2} x}{\partial t \partial b}(b, t)\right)= \\
& -\frac{\partial \phi}{\partial t}(b, t)\left(\int_{0}^{t} \exp \left(\int_{0}^{t^{\prime}} \phi\left(b, t^{\prime \prime}\right) \mathrm{d} t^{\prime \prime}\right) \mathrm{d} t^{\prime}+\frac{\partial x}{\partial b}(b, 0)\right)
\end{aligned}
$$

Since $\left.\exp \left(\int_{0}^{t} \phi\left(b, t^{\prime}\right) \mathrm{d} t^{\prime}\right) \frac{\partial^{2} x}{\partial t \partial b}(b, t)\right|_{t=0} \geq \epsilon$, and the right hand side of (A.3) is non-negative for $t \in\left[0, t_{1}\right]$, it follows that

$$
\left.\exp \left(\int_{0}^{t} \phi\left(b, t^{\prime}\right) \mathrm{d} t^{\prime}\right) \frac{\partial^{2} x}{\partial t \partial b}(b, t)\right|_{t=t_{1}} \geq \epsilon
$$

In the expression

$$
\frac{\partial \phi}{\partial t}(b, t)=\int_{-\infty}^{+\infty} g\left(b^{\prime}\right) \frac{\mathrm{d}^{2} f}{\mathrm{~d} x^{2}}\left(x\left(b^{\prime}, t\right)-x(b, t)\right)\left(\frac{\partial x}{\partial t}\left(b^{\prime}, t\right)-\frac{\partial x}{\partial t}(b, t)\right) \mathrm{d} b^{\prime}
$$

the factor $\frac{\mathrm{d}^{2} f}{\mathrm{~d} x^{2}}\left(x\left(b^{\prime}, t\right)-x(b, t)\right)$ has the opposite sign of $x\left(b^{\prime}, t\right)-x(b, t)$, which has the same sign as $b^{\prime}-b$ (since $\left.\frac{\partial x}{\partial t}(b, t) \geq 0, \forall b \in \mathbb{R}, \forall t \in \mathbb{R}^{+}\right)$. Since $\left|\frac{\partial x}{\partial t}\left(b^{\prime}, t\right)-\frac{\partial x}{\partial t}(b, t)-\left(b^{\prime}-b\right)\right| \leq 2 F$ it follows that $\left|\frac{\partial x}{\partial t}\left(b^{\prime}, t\right)-\frac{\partial x}{\partial t}(b, t)\right| \leq 2 F$ whenever $\frac{\partial x}{\partial t}\left(b^{\prime}, t\right)-\frac{\partial x}{\partial t}(b, t)$ and $b^{\prime}-b$ have different signs. As a consequence $\frac{\partial \phi}{\partial t}(b, t)$ is upper bounded by $2 F \sup \left(\frac{\mathrm{d}^{2} f}{\mathrm{~d} x^{2}}\right)$, and for any $\Delta t>0$ the right hand side of (A.3) is lower bounded for $t \in\left[t_{1}, t_{1}+\Delta t\right]$ (where the bound is independent of $b$ ). This implies the existence of a $\Delta t_{1}>0$ for which

$$
\exp \left(\int_{0}^{t} \phi\left(b, t^{\prime}\right) \mathrm{d} t^{\prime}\right) \frac{\partial^{2} x}{\partial t \partial b}(b, t) \geq 0
$$

for $t \in\left[t_{1}, t_{1}+\Delta t_{1}\right]$, for all $b \in \mathbb{R}$, and thus $\frac{\partial^{2} x}{\partial t \partial b}(b, t)$ is also non-negative for $t \in\left[0, t_{1}+\Delta t_{1}\right]$ (for all $b \in \mathbb{R}$ ), contradicting the definition of $t_{1}$. It follows that $\frac{\partial^{2} x}{\partial t \partial b}(b, t) \geq 0, \forall t \in \mathbb{R}^{+}, \forall b \in \mathbb{R}$.

\section{The intervals $\mathcal{I}_{k}$ and $\mathcal{S}$}

Since $\frac{\partial^{2} x}{\partial t \partial b}(b, t) \geq 0$ for $(b, t) \in \mathbb{R} \times \mathbb{R}^{+}$the expression $x\left(b_{2}, t\right)-x\left(b_{1}, t\right)$, for $b_{2}>b_{1}$, is non-decreasing in $t$. If it is bounded then the same holds for $x(b, t)-$ $x\left(b_{1}, t\right)$ and $x\left(b_{2}, t\right)-x(b, t)$ with $b \in\left(b_{1}, b_{2}\right)$ arbitrary. It follows that we can find intervals $\mathcal{I}_{k}\left(k \in I_{\mathcal{N}}\right.$, for some $\left.\mathcal{N} \in \mathbb{N} \cup\{+\infty\}\right)$ of non-zero length such that for any $k \in I_{\mathcal{N}}$ and $b_{1}, b_{2} \in \mathcal{I}_{k}$ the value of $x\left(b_{2}, t\right)-x\left(b_{1}, t\right)$ is bounded in $t$, and (since it is non-decreasing) approaches a constant for $t \rightarrow+\infty$. Let the intervals $\mathcal{I}_{k}$ be maximal, i.e. such that they cannot be extended, and let $\mathcal{N}$ also be maximal, i.e. such that for any pair $\left(b_{1}, b_{2}\right) \in \mathbb{R}^{2}$ with $b_{1} \neq b_{2}$ and $x\left(b_{2}, t\right)-x\left(b_{1}, t\right)$ bounded in $t, b_{1}$ and $b_{2}$ are contained in a common interval 
$\mathcal{I}_{k}$ for some $k \in I_{\mathcal{N}}$. Then $x$ exhibits clustering behavior with respect to the set of intervals $\mathcal{I}_{k}$.

Denote by $c_{k}$, resp. $d_{k}$, the lower, resp. upper, endpoint of the interval $\mathcal{I}_{k}$. Then for any $\epsilon>0$ and $b \in \mathcal{I}_{k}, x\left(d_{k}+\epsilon, t\right)-x(b, t)$ and $x(b, t)-x\left(c_{k}-\epsilon, t\right)$ are unbounded (and non-decreasing), and it is easy to verify that for $t \rightarrow+\infty$ the expression

$$
\begin{aligned}
& \left\langle\frac{\partial x}{\partial t}(b, t)\right\rangle_{\mathcal{I}_{k}}=\frac{1}{\alpha_{\mathcal{I}_{k}}} \int_{\mathcal{I}_{k}} \frac{\partial x}{\partial t}(b, t) g(b) \mathrm{d} b \\
& =\frac{1}{\alpha_{\mathcal{I}_{k}}} \int_{c_{k}}^{d_{k}} b g(b) \mathrm{d} b \\
& \quad+\frac{1}{\alpha_{\mathcal{I}_{k}}} \int_{c_{k}}^{d_{k}} \int_{-\infty}^{+\infty} g\left(b^{\prime}\right) f\left(x\left(b^{\prime}, t\right)-x(b, t)\right) g(b) \mathrm{d} b \\
& =\langle b\rangle_{\mathcal{I}_{k}}+\frac{1}{\alpha_{\mathcal{I}_{k}}} \int_{c_{k}}^{d_{k}} \int_{-\infty}^{c_{k}} g\left(b^{\prime}\right) f\left(x\left(b^{\prime}, t\right)-x(b, t)\right) g(b) \mathrm{d} b \\
& \quad+\frac{1}{\alpha_{\mathcal{I}_{k}}} \int_{c_{k}}^{d_{k}} \int_{d_{k}}^{+\infty} g\left(b^{\prime}\right) f\left(x\left(b^{\prime}, t\right)-x(b, t)\right) g(b) \mathrm{d} b
\end{aligned}
$$

will approach

$$
\langle b\rangle_{\mathcal{I}_{k}}-F \alpha_{\left(-\infty, c_{k}\right)}+F \alpha_{\left(d_{k},+\infty\right)},
$$

while, for any $b \in \mathcal{S} \triangleq \mathbb{R} \backslash \bigcup_{k \in I_{\mathcal{N}}} \mathcal{I}_{k}$,

$$
\lim _{t \rightarrow \infty} \frac{\partial x}{\partial t}(b, t)=b-F \alpha_{(-\infty, b)}+F \alpha_{(b,+\infty)}
$$

Defining $v$ by

$$
v(b)= \begin{cases}b-F \alpha_{(-\infty, b)}+F \alpha_{(b,+\infty)}, & b \in \mathcal{S} \\ \langle b\rangle_{\mathcal{I}_{k}}-F \alpha_{\left(-\infty, c_{k}\right)}+F \alpha_{\left(d_{k},+\infty\right)}, & b \in \mathcal{I}_{k}, \text { for some } k \in I_{\mathcal{N}},\end{cases}
$$

we obtain that $\lim _{t \rightarrow \infty} \frac{\partial x}{\partial t}(b, t)=v(b)$. Notice that, since $\frac{\partial x}{\partial t}(b, t)$ is nondecreasing in $b$, it follows that $v$ is also non-decreasing.

\section{The conditions (5)}

We now show that the intervals $\mathcal{I}_{k}$ and $\mathcal{S}$ satisfy (5). For any $p \in\left(c_{k}, d_{k}\right)$

$$
\begin{aligned}
&\left\langle\frac{\partial x}{\partial t}(b, t)\right\rangle_{\left(p, d_{k}\right)}-\left\langle\frac{\partial x}{\partial t}(b, t)\right\rangle_{\left(c_{k}, p\right)} \\
&=\langle b\rangle_{\left(p, d_{k}\right)}-\langle b\rangle_{\left(c_{k}, p\right)}-\left(\frac{1}{\alpha_{\left(p, d_{k}\right)}}+\frac{1}{\alpha_{\left(c_{k}, p\right)}}\right) \\
& \quad \times \int_{c_{k}}^{p} \mathrm{~d} b g(b) \int_{p}^{d_{k}} \mathrm{~d} b^{\prime} g\left(b^{\prime}\right) f\left(x\left(b^{\prime}, t\right)-x(b, t)\right)
\end{aligned}
$$


(using the bound $f\left(x\left(b^{\prime}, t\right)-x(b, t)\right) \leq F$ )

$$
\begin{aligned}
& \geq\langle b\rangle_{\left(p, d_{k}\right)}-\langle b\rangle_{\left(c_{k}, p\right)}-\left(\alpha_{\left(c_{k}, p\right)}+\alpha_{\left(p, d_{k}\right)}\right) F \\
& =\langle b\rangle_{\left(p, d_{k}\right)}-\langle b\rangle_{\left(c_{k}, p\right)}-\alpha_{\mathcal{I}_{k}} F
\end{aligned}
$$

and thus

$$
\langle b\rangle_{\left(p, d_{k}\right)}-\langle b\rangle_{\left(c_{k}, p\right)}-\alpha_{\mathcal{I}_{k}} F \leq \inf _{t \in \mathbb{R}}\left(\left\langle\frac{\partial x}{\partial t}(b, t)\right\rangle_{\left(p, d_{k}\right)}-\left\langle\frac{\partial x}{\partial t}(b, t)\right\rangle_{\left(c_{k}, p\right)}\right) .
$$

The right hand side cannot be negative since $\frac{\partial^{2} x}{\partial t \partial b}(b, t) \geq 0$. It can be verified not to be positive as follows. Pick $b_{1} \in\left(c_{k}, p\right)$ and $b_{2} \in\left(p, d_{k}\right)$ arbitrary. Then

$$
\begin{gathered}
\left\langle\frac{\partial x}{\partial t}(b, t)\right\rangle_{\left(p, d_{k}\right)}-\left\langle\frac{\partial x}{\partial t}(b, t)\right\rangle_{\left(c_{k}, p\right)} \\
=\frac{\alpha_{\left(p, b_{2}\right)}\left\langle\frac{\partial x}{\partial t}(b, t)\right\rangle_{\left(p, b_{2}\right)}+\alpha_{\left(b_{2}, d_{k}\right)}\left\langle\frac{\partial x}{\partial t}(b, t)\right\rangle_{\left(b_{2}, d_{k}\right)}}{\alpha_{\left(p, d_{k}\right)}} \\
\quad-\frac{\alpha_{\left(b_{1}, p\right)}\left\langle\frac{\partial x}{\partial t}(b, t)\right\rangle_{\left(b_{1}, p\right)}+\alpha_{\left(c_{k}, b_{1}\right)}\left\langle\frac{\partial x}{\partial t}(b, t)\right\rangle_{\left(c_{k}, b_{1}\right)}}{\alpha_{\left(c_{k}, p\right)}} \\
\leq \frac{\alpha_{\left(p, b_{2}\right)} \frac{\partial x}{\partial t}\left(b_{2}, t\right)+\left(d_{k}-b_{2}\right)(|b|+F)}{\alpha_{\left(p, d_{k}\right)}} \\
-\frac{\alpha_{\left(b_{1}, p\right)} \frac{\partial x}{\partial t}\left(b_{1}, t\right)-\left(b_{1}-c_{k}\right)(|b|+F)}{\alpha_{\left(c_{k}, p\right)}}
\end{gathered}
$$

since $\frac{\partial x}{\partial t}(b, t)$ is non-decreasing in $b$. It follows that

$$
\begin{aligned}
\inf _{t \in \mathbb{R}} & \left\langle\left\langle\frac{\partial x}{\partial t}(b, t)\right\rangle_{\left(p, d_{k}\right)}-\left\langle\frac{\partial x}{\partial t}(b, t)\right\rangle_{\left(c_{k}, p\right)}\right) \\
\leq & \frac{\alpha_{\left(p, b_{2}\right)}}{\alpha_{\left(p, d_{k}\right)}} v\left(b_{2}\right)-\frac{\alpha_{\left(b_{1}, p\right)}}{\alpha_{\left(c_{k}, p\right)}} v\left(b_{1}\right) \\
& \quad+(|b|+F)\left(\frac{\left(d_{k}-b_{2}\right)}{\alpha_{\left(p, d_{k}\right)}}+\frac{\left(b_{1}-c_{k}\right)}{\alpha_{\left(c_{k}, p\right)}}\right),
\end{aligned}
$$

which can be made arbitrarily small by letting $b_{1}$ and $b_{2}$ approach $c_{k}$ and $d_{k}$ respectively, since $v\left(b_{1}\right)=v\left(b_{2}\right)$. It follows that $\langle b\rangle_{\left(p, d_{k}\right)}-\langle b\rangle_{\left(c_{k}, p\right)}-\alpha_{\mathcal{I}_{k}} F \leq 0$, i.e. $(5 \mathrm{~d})$.

Taking the limits $p \gg c_{k}$ and $p \stackrel{\leq}{\rightarrow} d_{k}$ in $(5 \mathrm{~d})$ then results in $\langle b\rangle_{\left(c_{k}, d_{k}\right)} \leq$ $c_{k}+\alpha_{\mathcal{I}_{k}} F$ and $\langle b\rangle_{\left(c_{k}, d_{k}\right)} \geq d_{k}-\alpha_{\mathcal{I}_{k}} F$. From the fact that $v$ is non-decreasing (since $\frac{\partial x}{\partial t}(b, t)$ is non-decreasing in $b$ ), we obtain that

$$
\lim _{b \subseteq c_{k}} v(b) \leq \lim _{b \unlhd c_{k}} v(b)=\langle v\rangle_{\mathcal{I}_{k}}=\lim _{b \subseteq d_{k}} v(b) \leq \lim _{b \rightrightarrows d_{k}} v(b) .
$$

Combining these results leads to $\langle b\rangle_{\left(c_{k}, d_{k}\right)}=c_{k}+\alpha_{\mathcal{I}_{k}} F$ (i.e. (5b)), $\langle b\rangle_{\left(c_{k}, d_{k}\right)}=$ 
$d_{k}-\alpha_{\mathcal{I}_{k}} F($ i.e. $(5 \mathrm{c}))$, and

$$
\lim _{b \subseteq c_{k}} v(b)=\lim _{b \doteq c_{k}} v(b)=\langle v\rangle_{\mathcal{I}_{k}}=\lim _{b \subseteq d_{k}} v(b)=\lim _{b \unlhd d_{k}} v(b),
$$

proving that $v$ is continuous. Furthermore, since $v$ is non-decreasing in $\mathcal{S}$, it follows that $\frac{\partial v}{\partial b}(b)=1-2 F g(b) \geq 0, \forall b \in \mathcal{S}$ (i.e. (5a)), and the sets $\mathcal{I}_{k}$ and $\mathcal{S}$ satisfy the equations (5).

\section{B Persistence of the clustering conditions with increasing coupling strength}

In this section we provide some arguments for the explanation in section 4.3. We consider the growth of a single cluster with increasing coupling strength, and the merging process of two 'colliding' clusters, by investigating the conditions (5), implicitly assuming that these characterize the emerging clusters in the system (3) (see also the discussion below Theorem 4).

\section{B.1 Cluster growth}

We consider a set of intervals $\mathcal{I}_{k}\left(k \in I_{\mathcal{N}}\right)$ satisfying the conditions (5) (with $\left.\mathcal{S} \triangleq \mathbb{R} \backslash \bigcup_{k \in I_{\mathcal{N}}} \mathcal{I}_{k}\right)$, and we investigate the growth of these clusters when the coupling strength $F$ is increased.

Because of technical difficulties, our analysis may not cover all aspects that would be needed for a complete mathematical proof, and we will occasionally use a more heuristic approach, especially for particular distributions $g$ (see later on).

\section{B.1.1 Continuation of the cluster intervals}

For a particular interval $\mathcal{I}_{k^{\prime}}$, we investigate the continuation of $(6 \mathrm{~b})$, since this condition characterizes the cluster interval without relating it to the coupling strength $F$. After solving this equation one obtains a family of intervals for which (6a) can be used to calculate the corresponding values of the coupling strength.

Pick a $k^{\prime}$ in $I_{\mathcal{N}}$ and set $m_{0} \triangleq \frac{c_{k^{\prime}}+d_{k^{\prime}}}{2}, h_{0} \triangleq \frac{d_{k^{\prime}}-c_{k^{\prime}}}{2}, F_{0} \triangleq \frac{d_{k^{\prime}}-c_{k^{\prime}}}{2 \int_{c_{k^{\prime}}}^{d_{k^{\prime}} g\left(b^{\prime}\right) \mathrm{d} b^{\prime}}}$, and for $m \in \mathbb{R}$ and $h \in \mathbb{R}^{+}$, define $\mathcal{F}$ by

$$
\mathcal{F}(m, h) \triangleq\langle b\rangle_{(m-h, m+h)}-m \text {. }
$$


The equation $\mathcal{F}(m, h)=0$ relates the midpoint $m$ of an interval $\mathcal{I}_{k}$ to its half length $h$. If $g(m)>0$ then the function $\mathcal{F}$ is continuously differentiable for $h>0$ and along a solution of $\mathcal{F}(m, h)=0$ we obtain

$$
\begin{aligned}
\frac{\partial \mathcal{F}}{\partial m}(m, h)= & \frac{\partial}{\partial m}\left(\frac{\int_{m-h}^{m+h} b g(b) \mathrm{d} b}{\int_{m-h}^{m+h} g(b) \mathrm{d} b}-m\right) \\
= & \frac{(m+h) g(m+h)-(m-h) g(m-h)}{\int_{m-h}^{m+h} g(b) \mathrm{d} b} \\
& -\frac{(g(m+h)-g(m-h)) \int_{m-h}^{m+h} b g(b) \mathrm{d} b}{\left(\int_{m-h}^{m+h} g(b) \mathrm{d} b\right)^{2}}-1
\end{aligned}
$$

$\left(\mathcal{F}(m, h)=0 \Leftrightarrow \int_{m-h}^{m+h} b g(b) \mathrm{d} b=m \int_{m-h}^{m+h} g(b) \mathrm{d} b\right)$

$$
=\frac{h}{\int_{m-h}^{m+h} g(b) \mathrm{d} b}(g(m+h)+g(m-h))-1,
$$

and

$$
\frac{\partial \mathcal{F}}{\partial h}(m, h)=\frac{h}{\int_{m-h}^{m+h} g(b) \mathrm{d} b}(g(m+h)-g(m-h)),
$$

and thus (if $\frac{\partial \mathcal{F}}{\partial m}(m, h) \neq 0$ )

$$
\begin{aligned}
\frac{\mathrm{d} m}{\mathrm{~d} h}(h) & =-\frac{\frac{\partial \mathcal{F}}{\partial h}(m, h)}{\frac{\partial \mathcal{F}}{\partial m}(m, h)} \\
& =-\frac{g(m+h)-g(m-h)}{g(m+h)+g(m-h)-\frac{1}{h} \int_{m-h}^{m+h} g(b) \mathrm{d} b} .
\end{aligned}
$$

We will assume that $\frac{\partial \mathcal{F}}{\partial m}\left(m_{0}, h_{0}\right) \neq 0$, allowing us to apply the implicit function theorem to find a function $\tilde{m}:\left(h_{i}, h_{f}\right) \rightarrow \mathbb{R}: h \mapsto \tilde{m}(h)$, with $h_{0} \in\left(h_{i}, h_{f}\right)$ and $\mathcal{F}(\tilde{m}(h), h)=0$ for all $h \in\left(h_{i}, h_{f}\right)$. We assume that the interval $\left(h_{i}, h_{f}\right)$ is maximal.

Since the intervals $\mathcal{I}_{k}$ and $\mathcal{S}$ satisfy (5), the inequalities

$$
g(m \pm h) \leq \frac{1}{2 F}=\frac{1}{2 h} \int_{m-h}^{m+h} g\left(b^{\prime}\right) \mathrm{d} b^{\prime},
$$

are satisfied for $m=m_{0}, h=h_{0}$, and $F=F_{0}$, and $\frac{\partial \mathcal{F}}{\partial m}\left(m_{0}, h_{0}\right) \leq 0$, with equality only if the equation

$$
g(m-h)=g(m+h)=\frac{1}{2 h} \int_{m-h}^{m+h} g\left(b^{\prime}\right) \mathrm{d} b^{\prime}
$$

is satisfied for $m=m_{0}$ and $h=h_{0}$. 
Remark 7 If $h_{0}>0$, the case where (B.2) holds is only relevant for the system (3) if $g$ attains local maxima in both $m_{0}-h_{0}$ and $m_{0}+h_{0}$, since otherwise both $m_{0}-h_{0}$ and $m_{0}+h_{0}$ would belong to the interior of cluster intervals (associated with clusters that have emerged for smaller values of the coupling strength), and they cannot consitute the endpoints of an interval $\mathcal{I}_{k}$. Therefore, for a generic distribution $g$, the equation (B.2) is only relevant for the system (3) at the appearance of a cluster, when $h=0$. For convenience we will not elaborate on this.

For the values of $h \in\left(h_{i}, h_{f}\right)$ where (B.1) is satisfied with $m=\tilde{m}(h)$, it follows that

$$
\left|\frac{\mathrm{d} \tilde{m}}{\mathrm{~d} h}(h)\right| \leq 1
$$

and therefore $\tilde{c}_{k^{\prime}}(h) \triangleq \tilde{m}(h)-h \leq 0$ and $\tilde{d}_{k^{\prime}}(h) \triangleq \tilde{m}(h)+h \geq 0$, implying that $\tilde{F}(h) \triangleq \frac{h}{\int_{c_{k^{\prime}}(h)}^{d^{\prime}(h)} g\left(b^{\prime}\right) \mathrm{d} b^{\prime}} \geq 0$. Consequently the clusters grow with increasing coupling strength. As $h$ is increased from $h_{0}$ on, then, if one of the inequalities in (B.1) is no longer satisfied for some value of $h$ and $m=\tilde{m}(h)$, this implies (in the generic case, see also Remark 7) that another cluster must have appeared for a value of the coupling strength smaller than $\tilde{F}(h)$ at another local maximum of $g$, and that both clusters must have merged (see also section B.3) for an intermediate value of the coupling strength. This implies that the continuation of $\tilde{m}$ is no longer relevant for (3) if (B.1) is no longer satisfied.

On the other hand, assume that (B.1) is satisfied with $m=\tilde{m}(h)$, for all $h \in\left[h_{0}, h_{f}\right)$. Since then also $\left|\frac{\mathrm{d} \tilde{m}}{\mathrm{~d} h}(h)\right| \leq 1$, for $h \in\left[h_{0}, h_{f}\right)$, it follows that $m_{f} \triangleq \lim _{h \rightarrow h_{f}} \tilde{m}(h)$ exists (since any limit point $m_{f}$ satisfies $\tilde{m}(h)-\left(h_{f}-h\right) \leq$ $m_{f} \leq \tilde{m}(h)+\left(h_{f}-h\right)$ for any $\left.h \in\left[h_{0}, h_{f}\right)\right)$, and by the continuity of $\mathcal{F}$ it satisfies $\mathcal{F}\left(m_{f}, h_{f}\right)=0$. Furthermore, by the maximality of the interval $\left(h_{i}, h_{f}\right)$ as the domain of $\tilde{m}$ it follows that $\frac{\partial \mathcal{F}}{\partial m}\left(m_{f}, h_{f}\right)=0$. Since (B.1) is also satisfied for $m=m_{f}$ and $h=h_{f}$ (by continuity), both inequalities hold with equality, and, as noticed in Remark 7, this implies that $g$ is a non-generic distribution, and for concenience we will not elaborate on this. It follows that, for a generic distribution $g$, the growth of a cluster interval - as long as it is relevant for the system (3) - is characterized by the functions $\tilde{m}$ (or $\tilde{c}_{k}$ and $\tilde{d}_{k}$ ), together with $\tilde{F}$.

As noticed in section 4.3, the emergence of new clusters at values $b$ where a local maximum of $g(b)$ is attained when the coupling strength $F$ is increased above $1 /(2 g(b))$ ensures that (5a) remains satisfied. Furthermore, the evolution of the cluster intervals with $F$ according to the equations (6) ensures that $(5 \mathrm{~b})$ and $(5 \mathrm{c})$ are satisfied. In the next section we show that (5d) also remains satisfied. 


\section{B.2 The condition (5d)}

We consider the functions $\tilde{m}, \tilde{c}_{k^{\prime}}, \tilde{d}_{k^{\prime}}$, and $\tilde{F}$, introduced in the previous section, defined over an interval $\left(h_{i}, h_{f}\right]$ for which (B.1) holds with $m=\tilde{m}(h)$ for all $h$ in $\left(h_{i}, h_{f}\right]$. (Notice that the interval $\left(h_{i}, h_{f}\right]$ may no longer be the maximal interval in which $\tilde{m}$ can be defined.) This ensures that $\left|\frac{\mathrm{d} \tilde{m}}{\mathrm{~d} h}(h)\right| \leq 1$ and thus $\frac{\mathrm{d} \tilde{c}_{k^{\prime}}}{\mathrm{d} h}(h) \leq$ $0, \frac{\mathrm{d} \tilde{d}_{k^{\prime}}}{\mathrm{d} h}(h) \geq 0$ and $\frac{\mathrm{d} \tilde{F}}{\mathrm{~d} h}(h) \geq 0$ for $h$ in $\left(h_{i}, h_{f}\right]$. Pick a $p \in\left(\tilde{c}_{k^{\prime}}\left(h_{1}\right), \tilde{d}_{k^{\prime}}\left(h_{1}\right)\right)$, for some $h_{1} \in\left(h_{i}, h_{f}\right)$, and define the function $C$ by

$$
C(h) \triangleq\langle b\rangle_{\left(p, \tilde{d}_{k^{\prime}}(h)\right)}-\langle b\rangle_{\left(\tilde{c}_{k^{\prime}}(h), p\right)}-\alpha_{\left(\tilde{c}_{k^{\prime}}(h), \tilde{d}_{k^{\prime}}(h)\right)} \tilde{F}(h),
$$

for all $h$ in $\left[h_{1}, h_{f}\right]$. The function $C$ is differentiable in $\left[h_{1}, h_{f}\right]$, with

$$
\begin{aligned}
\frac{\mathrm{d} C}{\mathrm{~d} h}(h)= & \frac{\tilde{d}_{k^{\prime}}(h) g\left(\tilde{d}_{k^{\prime}}(h)\right) \frac{\mathrm{d} \tilde{d}_{k^{\prime}}}{\mathrm{d} h}(h)}{\alpha_{\left(p, \tilde{d}_{k^{\prime}}(h)\right)}(h)}-\frac{\langle b\rangle_{\left(p, \tilde{d}_{k^{\prime}}(h)\right)} g\left(\tilde{d}_{k^{\prime}}(h)\right) \frac{\mathrm{d} \tilde{d}_{k^{\prime}}}{\mathrm{d} h}(h)}{\alpha_{\left(p, \tilde{d}_{k^{\prime}}(h)\right)}} \\
& +\frac{\tilde{c}_{k^{\prime}}(h) g\left(\tilde{c}_{k^{\prime}}(h)\right) \frac{\mathrm{d} \tilde{c}_{k^{\prime}}}{\mathrm{d} h}(h)}{\alpha_{\left(\tilde{c}_{k^{\prime}}(h), p\right)}}-\frac{\langle b\rangle_{\left(\tilde{c}_{k^{\prime}}(h), p\right)} g\left(\tilde{c}_{k^{\prime}}(h)\right) \frac{\mathrm{d} \tilde{c}_{k^{\prime}}}{\mathrm{d} h}(h)}{\alpha_{\left(\tilde{c}_{k^{\prime}}(h), p\right)}} \\
& -\left(g\left(\tilde{d}_{k^{\prime}}(h)\right) \frac{\mathrm{d} \tilde{d}_{k^{\prime}}}{\mathrm{d} h}(h)-g\left(\tilde{c}_{k^{\prime}}(h)\right) \frac{\mathrm{d} \tilde{c}_{k^{\prime}}}{\mathrm{d} h}(h)\right) \tilde{F}(h) \\
\leq & \frac{g\left(\tilde{d}_{k^{\prime}}(h)\right) \frac{\mathrm{d} \tilde{d}_{k^{\prime}}}{\mathrm{d} h}(h)}{\alpha_{\left(p, \tilde{d}_{k^{\prime}}(h)\right)}\left(\tilde{d}_{k^{\prime}}(h)-\langle b\rangle_{\left(p, \tilde{d}_{k^{\prime}}(h)\right)}-\alpha_{\left(p, \tilde{d}_{k^{\prime}}(h)\right)} \tilde{F}(h)\right)} \\
& -\frac{g\left(\tilde{c}_{k^{\prime}}(h)\right) \frac{\mathrm{d} \tilde{c}_{k^{\prime}}}{\mathrm{d} h}(h)}{\alpha_{\left(\tilde{c}_{k^{\prime}}(h), p\right)}(h)}\left(-\tilde{c}_{k^{\prime}}(h)+\langle b\rangle_{\left(\tilde{c}_{k^{\prime}}(h), p\right)}-\alpha_{\left(\tilde{c}_{k^{\prime}}(h), p\right)} \tilde{F}(h)\right)
\end{aligned}
$$

(from $\mathcal{F}(\tilde{m}(h), h)=0$ and the definition of $\tilde{F}$ follow equivalent relations as in (5b) and (5c))

$$
\begin{aligned}
& =\frac{g\left(\tilde{d}_{k^{\prime}}(h)\right) \frac{\mathrm{d} \tilde{d}_{k^{\prime}}}{\mathrm{d} h}(h)}{\alpha_{\left(p, \tilde{d}_{k^{\prime}}(h)\right)}}\left(\langle b\rangle_{\left(\tilde{c}_{k^{\prime}}(h), \tilde{d}_{k^{\prime}}(h)\right)}+\alpha_{\left(\tilde{c}_{k^{\prime}}(h), \tilde{d}_{k^{\prime}}(h)\right)} \tilde{F}(h)-\langle b\rangle_{\left(p, \tilde{d}_{k^{\prime}}(h)\right)}\right. \\
& \left.\quad-\alpha_{\left(p, \tilde{d}_{k^{\prime}}(h)\right)} \tilde{F}(h)\right)-\frac{g\left(\tilde{c}_{k^{\prime}}(h)\right) \frac{\mathrm{d} \tilde{c}_{k^{\prime}}}{\mathrm{d} h}(h)}{\alpha_{\left(\tilde{c}_{k^{\prime}}(h), p\right)}}\left(\alpha_{\left(\tilde{c}_{k^{\prime}}(h), \tilde{d}_{k^{\prime}}(h)\right)} \tilde{F}(h)\right. \\
& \left.\quad-\langle b\rangle_{\left(\tilde{c}_{k^{\prime}}(h), \tilde{d}_{k^{\prime}}(h)\right)}+\langle b\rangle_{\left(\tilde{c}_{k^{\prime}}(h), p\right)}-\alpha_{\left(\tilde{c}_{k^{\prime}}(h), p\right)} \tilde{F}(h)\right) .
\end{aligned}
$$

Using the fact that $\alpha_{\left(\tilde{c}_{k^{\prime}}(h), p\right)}+\alpha_{\left(p, \tilde{d}_{k^{\prime}}(h)\right)}=\alpha_{\left(\tilde{c}_{k^{\prime}}(h), \tilde{d}_{k^{\prime}}(h)\right)}$ and

$$
\langle b\rangle_{\left(\tilde{c}_{k^{\prime}}(h), \tilde{d}_{k^{\prime}}(h)\right)}=\frac{\alpha_{\left(\tilde{c}_{k^{\prime}}(h), p\right)}\langle b\rangle_{\left(\tilde{c}_{k^{\prime}}(h), p\right)}+\alpha_{\left(p, \tilde{d}_{k^{\prime}}(h)\right)}\langle b\rangle_{\left(p, \tilde{d}_{k^{\prime}}(h)\right)}}{\alpha_{\left(\tilde{c}_{k^{\prime}}(h), \tilde{d}_{k^{\prime}}(h)\right)}}
$$


we can rewrite this in terms of $C(h)$ :

$$
\begin{aligned}
& \frac{\mathrm{d} C}{\mathrm{~d} h}(h) \leq \\
& \quad-\left(\frac{g\left(\tilde{d}_{k^{\prime}}(h)\right) \frac{\mathrm{d} \tilde{d}_{k^{\prime}}}{\mathrm{d} h}(h)}{\alpha_{\left(p, \tilde{d}_{k^{\prime}}(h)\right)}} \frac{\alpha_{\left(\tilde{c}_{k^{\prime}}(h), p\right)}}{\alpha_{\left(\tilde{c}_{k^{\prime}}(h), \tilde{d}_{k^{\prime}}(h)\right)}}-\frac{g\left(\tilde{c}_{k^{\prime}}(h)\right) \frac{\mathrm{d} \tilde{c}_{k^{\prime}}}{\mathrm{d} h}(h)}{\alpha_{\left(\tilde{c}_{k^{\prime}}(h), p\right)}} \frac{\alpha_{\left(p, \tilde{d}_{k^{\prime}}(h)\right)}}{\alpha_{\left(\tilde{c}_{k^{\prime}}(h), \tilde{d}_{k^{\prime}}(h)\right)}}\right) C(h) .
\end{aligned}
$$

It follows that if $C\left(h_{1}\right) \leq 0$, then $C(h) \leq 0$, for all $h$ in $\left[h_{1}, h_{f}\right]$.

As already mentioned in section 4.3 , the first occurrence of $(5 \mathrm{~d})$ for a given $p$ when increasing $F$ is automatically satisfied since then either $p=c_{k}$ or $p=d_{k}$ for some $k$ and thus (5d) follows from either (5b) or (5c). As we have just shown it will remain satisfied when the clusters continue to grow with further increases of $F$. (Notice however that the proof is not complete since it does not include the cases where $p=c_{k}$ or $p=d_{k}$, as the differentiability of the function $C$ is no longer clear in this case, and the notation should be extended. For convenience, we prefer not to elaborate on this.)

\section{B.3 Two clusters merging}

In this section we verify that two clusters, represented by the intervals $\mathcal{I}_{k}$ and $\mathcal{I}_{k+1}$, with $d_{k}-c_{k+1} \rightarrow 0$ (as a result of some limiting process $F \leq F^{\prime}$ for some $\left.F^{\prime}\right)$ and satisfying the equations $(5 \mathrm{~b}),(5 \mathrm{c})$ and $(5 \mathrm{~d})$, will result in a new cluster, with endpoints $c_{k}$ and $d_{k+1}$, and satisfying the same conditions. Since this merging process leaves $\mathcal{S}$ unchanged, the inequalities (5a) remain satisfied. For equations (5b) and (5c) we will consider the equivalent form (6).

It follows that

$$
\begin{aligned}
& \frac{\int_{c_{k}}^{d_{k+1}} g\left(b^{\prime}\right) \mathrm{d} b^{\prime}}{d_{k+1}-c_{k}} \\
& \quad=\frac{1}{d_{k+1}-c_{k}}\left(\int_{c_{k}}^{d_{k}} g\left(b^{\prime}\right) \mathrm{d} b^{\prime}+\int_{c_{k+1}}^{d_{k+1}} g\left(b^{\prime}\right) \mathrm{d} b^{\prime}\right) \\
& \quad=\frac{1}{d_{k+1}-c_{k}}\left(\frac{d_{k}-c_{k}}{2 F}+\frac{d_{k+1}-c_{k+1}}{2 F}\right) \\
& \quad=\frac{1}{2 F}
\end{aligned}
$$

and

$$
\begin{aligned}
\langle b\rangle_{\left(c_{k}, d_{k+1}\right)} & =\frac{1}{\alpha_{\left(c_{k}, d_{k+1}\right)}}\left(\alpha_{\left(c_{k}, d_{k}\right)}\langle b\rangle_{\left(c_{k}, d_{k}\right)}+\alpha_{\left(c_{k+1}, d_{k+1}\right)}\langle b\rangle_{\left(c_{k+1}, d_{k+1}\right)}\right) \\
& =\frac{1}{\frac{d_{k+1}-c_{k}}{2 F}}\left(\frac{\left(d_{k}-c_{k}\right)}{2 F} \frac{\left(c_{k}+d_{k}\right)}{2}+\frac{\left(d_{k+1}-c_{k+1}\right)}{2 F} \frac{\left(c_{k+1}+d_{k+1}\right)}{2}\right)
\end{aligned}
$$




$$
=\frac{d_{k+1}+c_{k}}{2}
$$

Concerning (5d) we consider the case where $p \in\left(c_{k}, d_{k}\right)$. The case $p \in$ $\left(c_{k+1}, d_{k+1}\right)$ is similar.

First notice that

$$
\begin{aligned}
\langle b\rangle_{\left(c_{k+1}, d_{k+1}\right)} & -\langle b\rangle_{\left(c_{k}, p\right)} \\
& =\langle b\rangle_{\left(c_{k}, d_{k}\right)}+\frac{d_{k+1}-c_{k}}{2}-\langle b\rangle_{\left(c_{k}, p\right)} \\
& =\frac{1}{\alpha_{\left(c_{k}, d_{k}\right)}}\left(\alpha_{\left(c_{k}, p\right)}\langle b\rangle_{\left(c_{k}, p\right)}+\alpha_{\left(p, d_{k}\right)}\langle b\rangle_{\left(p, d_{k}\right)}\right)+\alpha_{\left(c_{k}, d_{k+1}\right)} F-\langle b\rangle_{\left(c_{k}, p\right)} \\
& =\frac{\alpha_{\left(p, d_{k}\right)}}{\alpha_{\left(c_{k}, d_{k}\right)}}\left(\langle b\rangle_{\left(p, d_{k}\right)}-\langle b\rangle_{\left(c_{k}, p\right)}\right)+\alpha_{\left(c_{k}, d_{k+1}\right)} F
\end{aligned}
$$

Using this equation, it follows that

$$
\begin{aligned}
\langle b\rangle_{\left(p, d_{k+1}\right)} & -\langle b\rangle_{\left(c_{k}, p\right)} \\
& =\frac{\left(\alpha_{\left(p, d_{k}\right)}\langle b\rangle_{\left(p, d_{k}\right)}+\alpha_{\left(c_{k+1}, d_{k+1}\right)}\langle b\rangle_{\left(c_{k+1}, d_{k+1}\right)}\right)-\left(\alpha_{\left(p, d_{k}\right)}+\alpha_{\left(c_{k+1}, d_{k+1}\right)}\right)\langle b\rangle_{\left(c_{k}, p\right)}}{\alpha_{\left(p, d_{k+1}\right)}} \\
& =\frac{\alpha_{\left(p, d_{k}\right)}\left(\langle b\rangle_{\left(p, d_{k}\right)}-\langle b\rangle_{\left(c_{k}, p\right)}\right)+\alpha_{\left(c_{k+1}, d_{k+1}\right)}\left(\langle b\rangle_{\left(c_{k+1}, d_{k+1}\right)}-\langle b\rangle_{\left(c_{k}, p\right)}\right)}{\alpha_{\left(p, d_{k+1}\right)}} \\
& =\frac{\alpha_{\left(p, d_{k}\right)}}{\alpha_{\left(p, d_{k+1}\right)}} \frac{\alpha_{\left(c_{k}, d_{k+1}\right)}}{\alpha_{\left(c_{k}, d_{k}\right)}}\left(\langle b\rangle_{\left(p, d_{k}\right)}-\langle b\rangle_{\left(c_{k}, p\right)}\right)+\frac{\alpha_{\left(c_{k}, d_{k+1}\right)}}{\alpha_{\left(p, d_{k+1}\right)}} \alpha_{\left(c_{k+1}, d_{k+1}\right)} F \\
& \leq \alpha_{\left(c_{k}, d_{k+1}\right)} F .
\end{aligned}
$$

\section{References}

[1] D. Aeyels and F. De Smet. A model for the dynamical process of cluster formation. In 7th IFAC Symposium on Nonlinear Control Systems (NOLCOS 2007), pages 260-264, August 2007.

[2] D. Aeyels and F. De Smet. A mathematical model for the dynamics of clustering. Physica D: Nonlinear Phenomena, 273(19):2517-2530, October 2008 .

[3] D. Aeyels and J. A. Rogge. Existence of partial entrainment and stability of phase locking behavior of coupled oscillators. Progress of Theoretical Physics, 112(6):921-942, December 2004.

[4] L. Basnarkov and V. Urumov. Phase transitions in the Kuramoto model. Physical Review E, 76:057201, 2007.

[5] C. M. Bordogna and E. V. Albano. Statistical methods applied to the study of opinion formation models: a brief overview and results of a nu- 
merical study of a model based on the social impact theory. Journal of Physics: Condensed Matter, 19(6):065144 (16pp), February 2007.

[6] A. Czirók, E. Ben-Jacob, I. Cohen, and T. Vicsek. Formation of complex bacterial colonies via self-generated vortices. Physical Review E, 54(2):1791-1801, August 1996.

[7] F. De Smet and D. Aeyels. Resonances and entrainment breakup in Kuramoto models with multimodal frequency densities. Physical Review E (Statistical, Nonlinear, and Soft Matter Physics), 77(6):066212, 2008.

[8] F. De Smet and D. Aeyels. Clustering in a network of non-identical and mutually interacting agents. Proceedings of the Royal Society A, 465:745768, March 2009.

[9] G. Deffuant, F. Amblard, G. Weisbuch, and T. Faure. How can extremism prevail? a study based on the relative agreement interaction model. Journal of Artificial Societies and Social Simulation, 5(4), 2002.

[10] D. Golomb, D. Hansel, B. Shraiman, and H. Sompolinsky. Clustering in globally coupled phase oscillators. Physical Review A, 45(6):3516-3530, March 1992.

[11] G. Grégoire, H. Chaté, and Y. Tu. Moving and staying together without a leader. Physica D, 181(3-4):157-170, July 2003.

[12] R. Hegselmann and U. Krause. Opinion dynamics and bounded confidence: models, analysis and simulation. Journal of Artificial Societies and Social Simulation, 5(3), 2002.

[13] C. Huepe and M. Aldana. Intermittency and clustering in a system of self-driven particles. Phys. Rev. Lett., 92(16):168701, Apr 2004.

[14] Y. Kuramoto. Cooperative dynamics of oscillator community. Supplement of the Progress of Theoretical Physics, 79:223-240, 1984.

[15] Y. Maistrenko, O. Popovych, O. Burylko, and P. A. Tass. Mechanism of desynchronization in the finite-dimensional Kuramoto model. Physical Review Letters, 93(8):084102, August 2004.

[16] S. C. Manrubia, A. S. Mikhailov, and D. H. Zanette. Emergence of Dynamical Order: Synchronization Phenomena in Complex Systems, volume 2 of World Scientific Lecture Notes in Complex Systems. World Scientific, 2004.

[17] A. S. Mikhailov and D. H. Zanette. Noise-induced breakdown of coherent collective motion in swarms. Physical Review E, 60(4):4571+, October 1999.

[18] L. G. Morelli, H. A. Cerdeira, and D. H. Zanette. Frequency clustering of coupled phase oscillators on small-world networks. European Physical Journal B, 43:243-250, Jan. 2005.

[19] K. Okuda. Variety and generality of clustering in globally coupled oscillators. Physica D, 63(3-4):424-436, 1993.

[20] D. Pazó. Thermodynamic limit of the first-order phase transition in the Kuramoto model. Physical Review E, 72:046211, 2005.

[21] F. Peruani, A. Deutsch, and M. Bär. Nonequilibrium clustering of selfpropelled rods. Phys. Rev. E, 74(3):030904, Sep 2006. 
[22] A. Pluchino, V. Latora, and A. Rapisarda. Changing opinions in a changing world: a new perspective in sociophysics. International Journal of Modern Physics C, 16(4):515-531, April 2005.

[23] C. W. Reynolds. Flocks, herds, and schools: A distributed behavioral model. Computer Graphics, 21(4):25-34, 1987. (SIGGRAPH '87 Conference Proceedings).

[24] S. H. Strogatz. From Kuramoto to Crawford: exploring the onset of synchronization in populations of coupled oscillators. Physica D, 143:120, 2000.

[25] S. H. Strogatz. SYNC: The Emerging Science of Spontaneous Order. Hyperion Press, New York, 2003.

[26] K. Sznajd-Weron and J. Sznajd. Opinion evolution in closed community. International Journal of Modern Physics C, 11(6):1157-1165, September 2000.

[27] P. Tass. Phase and frequency shifts in a population of phase oscillators. Physical Review E, 56(2):2043-2060, August 1997. 